\title{
STRUCTURE MAPPINGS, COEXTENSIONS AND REGULAR FOUR-SPIRAL SEMIGROUPS
}

\author{
BY \\ JOHN MEAKIN ${ }^{1}$
}

\begin{abstract}
The structure mapping approach to regular semigroups developed by K. S. S. Nambooripad and J. Meakin is used to describe the $\mathcal{C}$-coextensions of the fundamental four-sprial semigroup and hence to describe the structure of all regular semigroups whose idempotents form a four-spiral biordered set. Isomorphisms between regular four-spiral semigroups are studied. The notion of structural uniformity of a regular semigroup is defined and exploited.
\end{abstract}

There is a standard strategy which has been used by many authors in recent years to describe the structure of a prescribed class $C$ of regular semigroups. This strategy involves two major steps: first, build the fundamental semigroups in the class $\mathcal{C}$, the second, build an arbitrary semigroup in class $\mathcal{C}$ as an $\mathcal{H}$-coextension of one of the fundamental semigroups in $\mathcal{C}$. A regular semigroup $S$ is called fundamental if the only idempotent-separating congruence on $S$ is the identity congruence: $S$ is called an $\mathcal{H}$-coextension of $T$ if there is an idempotent-separating homomorphism $\theta$ mapping $S$ onto $T$.

Pioneering work in the development of this strategy was done by W. D. Munn [15], [16] who described the structure of fundamental inverse semigroups: he explicitly proposed the above strategy in [17] and numerous authors have followed his approach. Fundamental orthodox semigroups were described by T. E. Hall [8] and fundamental regular semigroups have been described by K. S. S. Nambooripad [18], [19], T. E. Hall [9], A. H. Clifford [2], and by P. A. Grillet [6] (who calls them reduced). $\mathcal{H}$-coextensions of regular and inverse semigroups have been studied by a large number of authors: we refer the reader to the papers by Leech [11], Coudron [4], D'Alarcao [5], Grillet [7] and Meakin [14], but this is by no means a complete list.

In the present paper we adopt this strategy to describe the structure of all regular semigroups whose idempotents form the four-spiral biordered set $E_{4}$

Received by the editors July 15, 1977.

AMS (MOS) subject classifications (1970). Primary $20 \mathrm{M} 10$.

Key words and phrases. Regular semigroup, structure mappings, $\mathcal{H}$-coextensions, structural uniformity, four-spiral semigroup, biordered set, bisimple w-semigroup, trace, Rees groupoid, natural partial order on a regular semigroup, pseudo-semilattice, pseudo-inverse semigroup.

${ }^{1}$ This research was supported by NSF Grant No. MCS77-02829. 
introduced by K. Byleen, F. Pastijn and the present author in [1]. In [1], the authors described the structure of the fundamental four-spiral semigroup $\mathrm{Sp}_{4}$ (a basic building block for bisimple idempotent-generated semigroups which are not completely simple). We approach the problem of describing the $\mathcal{K}$-coextensions of $\mathrm{Sp}_{4}$ by means of the structure mappings on a regular semigroup $S$ : the structure mappings on $S$ are a family of mappings between $\Re$-classes and between $\mathcal{L}$-classes of $S$ which reduce all products in $S$ to products in the trace of $S$-we refer the reader to the papers by Nambooripad [18], [20] and the present author [12], [13] for a description of the structure mappings.

In [14] the author studied $\mathcal{H}$-coextensions of inverse semigroups from the structure mapping approach. Many of these ideas and results are extended to the regular case in the present work: in particular the notion of "structural uniformity" is defined for regular semigroups and is heavily exploited to build the $\mathcal{K}$-coextensions of the fundamental four-spiral. The resulting theorem is analogous to Reilly's theorem [23] on the structure of bisimple $\omega$-semigroups.

1. Preliminary results, notation. We assume that the reader is familiar with the basic ideas and results of semigroup theory as presented in the book of Clifford and Preston [3]. In addition we assume familiarity with Nambooripad's notion [18], [19] of a biordered set $\left(E, \omega^{r}, \omega^{l}, \tau\right)$ and we shall use his notation and results extensively without comment.

By the trace of a regular semigroup $S$ we mean the partial groupoid $\operatorname{tr}(S)=(S, \cdot)$ with partial binary operation $a \cdot b$ defined (and equal to $a b$ ) if and only if there is an idempotent $e=e^{2}$ in $L_{a} \cap R_{b}: \operatorname{tr}(S)$ is clearly a disjoint union of Rees groupoids, whose structure is known from the Rees theorem ([3, Theorem 3.5]). (A Rees groupoid is the partial groupoid $M=$ $\Re^{0}(G ; I, \Lambda ; P) \backslash\{0\}$ obtained from the Rees matrix semigroup $M^{0}=$ $\mathfrak{N}^{0}(G ; I, \Lambda ; P)$ by removing the 0 and leaving undefined all products in $M^{0}$ which were previously defined to be 0 .)

The trace of a regular semigroup $S$ carries a certain part of the structure of $S$ and the remaining part of the structure is determined by the "structure mappings" on $S$ which we shall now define. Let $\kappa=\omega^{r} \cup \omega^{l}$ and for idempotents $e, f \in E(S)$ with $f \kappa e$ define mappings $\phi_{e, f}: R_{e} \rightarrow R_{f}$ and $\psi_{e, f}$ : $L_{e} \rightarrow L_{f}$ as follows:

$$
x \phi_{e, f}=f x \quad \forall x \in R_{e} \quad \text { and } y \psi_{e, f}=y f \quad \forall y \in L_{e} .
$$

The mappings

$$
\Phi=\left\{\phi_{e, f}: R_{e} \rightarrow R_{f} \mid f \kappa e\right\} \quad \text { and } \Psi=\left\{\psi_{e,}: L_{e} \rightarrow L_{f} \mid f \kappa e\right\}
$$

are called the structure mappings on $S$. These mappings may be used to reduce all products in $S$ to products in $\operatorname{tr}(S)$ as follows: for $a, b \in S$ we have

$$
a b=\left(a \psi_{e, h}\right) \cdot\left(b \phi_{f, h}\right)
$$


where $e=e^{2}$ is an idempotent in $L_{a}, f=f^{2}$ is an idempotent in $R_{b}$ and $h=h^{2}$ is an element of the sandwich set $S(e, f)$ of $e$ and $f$. The product on the right-hand side of (1) is a product in $\operatorname{tr}(S)$ and is independent of the choice of $e=e^{2} \in L_{a}$ and $f=f^{2} \in R_{b}$ since $S(e, f)=S\left(e^{\prime}, f^{\prime}\right)$ if $e \mathcal{L} e^{\prime}$ and $f \Re f^{\prime}$.

Conditions which the set $\Phi \cup \Psi$ of mappings must satisfy are discussed in [13]: if we start with a set $\mathscr{N}=\left\{M_{\alpha} \mid \alpha \in J\right\}$ of mutually disjoint Rees groupoids $M_{\alpha}$, equip the idempotents of $\cup_{\alpha \in J} M_{\alpha}$ with a partial regular band structure and take any family $\Phi \cup \Psi$ of mappings which satisfy these conditions, then the multiplication (1) turns $\cup_{\alpha \in J} M_{\alpha}$ into a regular semigroup which we denote by $S(\Re, \Phi, \Psi)$. (This was denoted by $S(\Phi, \Psi)$ in [13].) For a discussion of this, and its connection with Nambooripad's approach [20], we refer the reader to the author's paper [13]. We shall consistently use the notation $S(\mathfrak{N}, \Phi, \Psi)$ for the regular semigroup with trace $\cup_{\alpha \in J} M_{\alpha}\left(\mathscr{N}=\left\{M_{\alpha} \mid \alpha \in J\right\}\right)$ and set $\Phi \cup \Psi$ of structure mappings.

Nonzero elements of the Rees matrix semigroup $M^{0}=\mathscr{N}^{0}(G ; I, \Lambda ; P)$ will be denoted by triples $(i, g, \lambda) \in I \times G \times \Lambda$ :

$$
R_{i}=\{(i, g, \lambda) \mid g \in G, \lambda \in \Lambda\}
$$

denotes the $R$-class containing $(i, g, \lambda), L_{\lambda}$ denotes the corresponding $\mathcal{L}$-class and $H_{i \lambda}=R_{i} \cap L_{\lambda}$ is the corresponding $\mathcal{H}$-class. If $\mathscr{N}=\left\{M_{\alpha} \mid \alpha \in J\right\}$ is a set of mutually disjoint Rees groupoids

$$
M_{\alpha}=\mathfrak{N}^{0}\left(G_{\alpha} ; I_{\alpha}, \Lambda_{\alpha} ; P_{\alpha}\right) \backslash\left\{0_{\alpha}\right\}
$$

we shall denote the identity of $G_{\alpha}$ by $1_{\alpha}$ and the set $\left\{(i, \lambda) \in I_{\alpha} \times \Lambda_{\alpha} \mid p_{\lambda i} \neq\right.$ $0\}$ by $X_{\alpha}$ : thus $X_{\alpha}=\left\{(i, \lambda) \in I_{\alpha} \times \Lambda_{\alpha} \mid H_{i \lambda}\right.$ contains an idempotent $\}$. We denote $\cup_{\alpha \in J} I_{\alpha}$ by $I$ and $\cup_{\alpha \in J} \Lambda_{\alpha}$ by $\Lambda$. For $(i, \lambda) \in X_{\alpha}$ (for some $\alpha \in J$ ), the idempotent in $H_{i \lambda}$ is, of course, $\left(i, p_{\lambda i}^{-1}, \lambda\right)$ : we shall of ten abbreviate this to $(i, \lambda)$ if no confusion can arise. Thus if for some $(i, \lambda) \in X_{\alpha}$ and $(j, \mu) \in$ $X_{\beta}$ we have $\left(j, p_{\mu j}^{-1}, \mu\right) \kappa\left(i, p_{\lambda i}^{-1}, \lambda\right)$ we shall simply write $(j, \mu) \kappa(i, \lambda)$. We denote the sandwich set of $(i, \lambda)$ and $(j, \mu)$ by $S(\lambda, j)$ since it depends only on $L_{\lambda}$ and $R_{j}$ and not on $i$ or $\mu$. Let $S=S(\mathscr{N}, \Phi, \Psi)$ be a regular semigroup (with $\mathscr{R}=\left\{M_{\alpha} \mid \alpha \in J\right\}$ as above) and suppose that $(j, \mu) \kappa(i, \lambda)$ for some $(j, \mu) \in X_{\beta},(i, \lambda) \in X_{\alpha}$ : we denote the structure mapping $\phi_{(i, \lambda),(j, \mu)}: R_{i} \rightarrow R_{j}$ by $\phi_{i, j, \mu}$ (since it is independent of $\lambda$ ); similarly $\psi_{\lambda, \mu, j}$ denotes the corresponding structure mapping from $L_{\lambda}$ to $L_{\mu}$. The mapping $\phi_{i, j, \mu}$ is defined only if $\exists \alpha, \beta \in J$ and $\lambda \in \Lambda$ such that $(j, \mu) \in X_{\beta},(i, \lambda) \in X_{\alpha}$ and $(j, \mu) \kappa(i, \lambda)$ : a similar comment applies to $\psi_{\lambda, \mu, j}$.

Let $(i, g, \lambda),(j, h, \mu) \in S$ and let $(k, \nu) \in S(\lambda, j)$ : then $L_{\nu} \leqslant L_{\lambda}$ (i.e., $S L_{\nu} \subseteq$ $S L_{\lambda}$ ) and $R_{k}<R_{j}$ (i.e., $R_{k} S \subseteq R_{j} S$ ) and so there are structure mappings $\phi_{j, k, \nu}: R_{j} \rightarrow R_{k}$ and $\psi_{\lambda, v, k}: L_{\lambda} \rightarrow L_{\nu}$ and the multiplication (1) may be rewritten 
in the form

$$
(i, g, \lambda)(j, h, \mu)=(i, g, \lambda) \psi_{\lambda, \nu, k}(j, h, \mu) \phi_{j, k, v} .
$$

We shall frequently use this form of the multiplication in a regular semigroup in the sequel.

In [22], Nambooripad introduced a natural partial order on a regular semigroup $S$. This order is defined by $x<y$ iff $R_{x}<R_{y}$ and $x=f y$ for some $f \in E\left(R_{x}\right)$. We reformulate Nambooripad's Proposition 1.2 [22] in the present notation.

Proposition 1.1 (NAmbooripad). If $(i, g, \lambda)$ and $(j, h, \mu)$ belong to the regular semigroup $S=S(\Re, \Phi, \Psi)$, the following statements are equivalent:

(a) $(j, h, \mu)<(i, g, \lambda)$;

(b) $R_{j}<R_{i}$ and there exists $\nu \in \Lambda$ such that

$$
(i, g, \lambda) \phi_{i, j, \nu}=(j, h, \mu)
$$

(c) $L_{\mu}<L_{\lambda}$ and there exists $k \in I$ such that

$$
(i, g, \lambda) \psi_{\lambda, \mu, k}=(j, h, \mu)
$$

ReMarK 1.2. Condition (b) guarantees that there exist $\alpha, \beta \in J$ and $\rho \in \Lambda$ such that $(i, \rho) \in X_{\alpha},(j, \nu) \in X_{\beta}$ and $f=(j, \nu) \omega^{r}(i, \rho)=e$ : the mapping $\phi_{i, j, \nu}$ is then the same as $\phi_{e, f}$. Since $f e$ is an idempotent in $R_{f}$ for which fe $\omega e$ and since $\phi_{e, f}=\phi_{e, f e}$ it follows that Nambooripad's condition (b) of his Proposition 1.2 [22] is satisfied: a similar comment above shows that our condition (c) above is equivalent to Nambooripad's condition (c) in his theorem.

If $S=S(\mathfrak{T}, \Phi, \Psi)$ and $S^{\prime}=S\left(\mathscr{N} \mathcal{L}^{\prime}, \Phi^{\prime}, \Psi^{\prime}\right)$ are regular semigroups and $\theta$ : $S \rightarrow S^{\prime}$ is a mapping from $S$ onto $S^{\prime}$ which preserves and reflects Green's $\Re$ and $\mathcal{L}$ relations (i.e., $a \theta \rho b \theta$ in $S^{\prime}$ iff $a \rho b$ in $S$ for $\rho \in\{\Re, \mathcal{L}\}$ ) then $\theta$ also preserves and reflects Green's $\mathcal{H}$ and $\mathscr{D}$ relations and so we may write $\mathscr{R}=\left\{M_{\alpha} \mid \alpha \in J\right\}$ and $\Re^{\prime}=\left\{M_{\alpha}^{\prime} \mid \alpha \in J\right\}$ where

$$
M_{\alpha}=\Re^{0}\left(G_{\alpha} ; I_{\alpha}, \Lambda_{\alpha} ; P_{\alpha}\right) \backslash\left\{0_{\alpha}\right\}
$$

and

$$
M_{\alpha}^{\prime}=\mathscr{T}^{0}\left(G_{\alpha}^{\prime} ; I_{\alpha}, \Lambda_{\alpha} ; P_{\alpha}^{\prime}\right) \backslash\left\{0_{\alpha}\right\},
$$

and we may assume that for each $i \in I_{\alpha}, \lambda \in \Lambda_{\alpha}, g \in G_{\alpha}$ (and $\alpha \in J$ ), there exists $g^{\prime} \in G_{\alpha}^{\prime}$ such that $(i, g, \lambda) \theta=\left(i, g^{\prime}, \lambda\right)$. With this notation it follows that for $(i, \lambda) \in X_{\alpha}=X_{\alpha}^{\prime}$ and $(j, \mu) \in X_{\beta}=X_{\beta}^{\prime},(j, \mu) \kappa(i, \lambda)$ in $S$ iff $(j, \mu) \kappa^{\prime}(i, \lambda)$ in $S^{\prime}$ : the corresponding structure mapping in $S$ is denoted by $\phi_{i, j, \mu}\left[\psi_{\lambda, \mu, j}\right]$ and that in $S^{\prime}$ by $\phi_{i, j, \mu}^{\prime}\left[\psi_{\lambda, \mu, j}^{\prime}\right]$. With this notational convention in mind we prove the following theorem (cf. [14, Theorem 1.5]). 
THEOREM 1.3. Let $\theta$ be a mapping from $S=S(\mathfrak{T}, \Phi, \Psi)$ onto $S^{\prime}=$ $S\left(\Re{ }^{\prime}, \Phi^{\prime}, \Psi^{\prime}\right)$ which preserves and reflects Green's $R$ and $\mathcal{L}$ relations. Then $\theta$ is an idempotent-separating homomorphism from $S$ onto $S^{\prime}$ iff both

(a) $\theta$ is a (partial groupoid) homomorphism from $\operatorname{tr}(S)$ onto $\operatorname{tr}\left(S^{\prime}\right)$, and

(b) $\theta$ commutes with all the structure mappings; i.e., for $(i, \lambda) \in X_{\alpha}$ and $(j, \mu) \in X_{\beta}$ with $(j, \mu) \kappa(i, \lambda)$ the diagrams

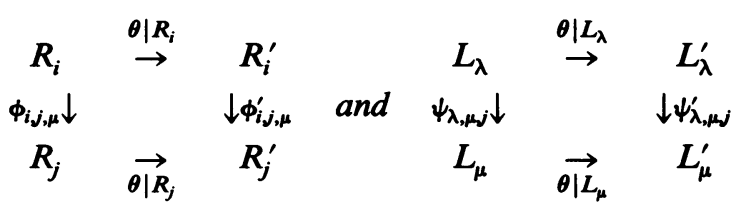

all commute.

Proof. Suppose first that $\theta$ is an idempotent-separating homomorphism from $S$ onto $S^{\prime}: \theta$ clearly satisfies condition (a). If $f=(j, \mu) \kappa(i, \lambda)=e$ and $a \in R_{e}$, then

$$
a \phi_{i, j, \mu} \theta=a \phi_{e_{j} f} \theta=(f a) \theta=(f \theta)(a \theta)=(a \theta) \phi_{i, j, \mu}^{\prime} ;
$$

a similar argument shows that $\psi_{\lambda, \mu, j} \theta=\theta \psi_{\lambda, \mu, j}^{\prime}$.

Suppose conversely that $\theta$ satisfies conditions (a) and (b). Let $(i, g, \lambda)$, $(j, h, \mu) \in S$ and let $(k, \nu) \in S(\lambda, j)$ : then by (2),

$$
\begin{aligned}
{[(i, g, \lambda)(j, h, \mu)] \theta } & =\left[(i, g, \lambda) \psi_{\lambda, \nu, k}(j, h, \mu) \phi_{j, k, \nu}\right] \theta \\
& =\left[(i, g, \lambda) \psi_{\lambda, \nu, k} \theta\right]\left[(j, h, \mu) \phi_{j, k, \nu} \theta\right] \\
& \text { by condition (a) } \\
& =\left[(i, g, \lambda) \theta \psi_{\lambda, \nu, k}^{\prime}\right]\left[(j, h, \mu) \theta \phi_{j, k, \nu}^{\prime}\right] \\
& =[(i, g, \lambda) \theta][(j, h, \mu) \theta] \text { by condition (b) }
\end{aligned}
$$

so $\theta$ is a homomorphism: $\theta$ is idempotent-separating since it preserves and

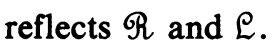

REMARK 1.4. The hypothesis (b) of Theorem 1.3 may be weakened slightly. If $(k, \nu) \in S(\lambda, j)$ then in fact $R_{k} \leqslant R_{j}$ and $L_{\nu} \leqslant L_{\lambda}$, so it follows that we only require $\theta$ to commute with the structure mappings $\phi_{i, j, \mu}$ for $R_{j}<R_{i}$ and $\psi_{\lambda, \mu, j}$ for $L_{\mu}<L_{\lambda}$. We remark at this stage that Nambooripad [20] uses only the structure mappings $\phi_{e, f}: R_{e} \rightarrow R_{f}$ for $f \omega^{r} e$ and $\psi_{e, f}: L_{e} \rightarrow L_{f}$ for $f \omega^{l} e$ (i.e., he does not use the mappings $\phi_{e, f}$ for $f \omega^{l} e$ and $\psi_{e, f}$ for $f \omega^{r} e$ ). There are certain advantages and certain disadvantages to using all of the structure mappings $\phi_{e, f}, \psi_{e, f}$ for $f \kappa e$ as we have done here (see [13] for a discussion of this). We make the general remark here that all of our results in the present paper hold true if we interpret the structure mappings in Nambooripad's sense, as the reader can easily verify by checking the proofs. Some of our results apply only 
to Nambooripad's structure mappings (e.g., Proposition 1.1): in these cases the situation is made clear in the statement of the result.

We obtain a number of immediate corollaries of Theorem 1.3.

Corollary 1.5. Let $\theta$ be a bijection from $S=S(\Re, \Phi, \Psi)$ onto $S^{\prime}=$ $S\left(\mathfrak{N}{ }^{\prime}, \Phi^{\prime}, \Psi^{\prime}\right)$. Then $\theta$ is an isomorphism iff

(a) $\theta$ is a trace isomorphism from $\operatorname{tr}(S)$ onto $\operatorname{tr}\left(S^{\prime}\right)$, and

(b) $\theta$ commutes with all the structure mappings.

COROLlaRY 1.6. Let $\theta$ be a bijection from $S=S(\Re, \Phi, \Psi)$ onto $S^{\prime}=$ $S\left(\mathfrak{T} \mathfrak{R}^{\prime}, \Phi^{\prime}, \Psi^{\prime}\right)$. Denote the natural partial order on $S\left[S^{\prime}\right]$ by $<\left[<^{\prime}\right]$. Then $\theta$ is an isomorphism iff

(a) $\theta$ is a trace isomorphism from $\operatorname{tr}(S)$ onto $\operatorname{tr}\left(S^{\prime}\right)$, and

(b) $\theta$ is an isomorphism from the poset $(S, \leqslant)$ onto the poset $\left(S^{\prime},<^{\prime}\right)$.

Proof. This follows from Corollary 1.5, Remark 1.4 and Proposition 1.1 and the fact that $\theta^{-1}$ satisfies (a) and (b) of Corollary 1.5.

COROLlaRY 1.7. Green's relation $\mathcal{H}$ is a congruence on $S=S(\mathfrak{T}, \Phi, \Psi)$ iff all of the structure mappings are $\mathcal{K}$-class preserving; i.e., iff $(i, g, \lambda) \phi_{i, j, \mu} \mathcal{H}$ $(i, h, \lambda) \phi_{i, j, \mu}$ and $(i, g, \lambda) \psi_{\lambda, \mu, j} \mathcal{H}(i, h, \lambda) \psi_{\lambda, \mu, j}$ whenever $g, h \in G_{\alpha}, i \in I_{\alpha}, \lambda$ $\in \Lambda_{\alpha}$ (for some $\alpha \in J$ ) and the mappings $\phi_{i, j, \mu}$ and $\psi_{\lambda, \mu, j}$ are defined.

Proof. Suppose first that $\mathcal{H}$ is a congruence on $S$, so that $S / \mathcal{H}$ is combinatorial, and denote the element $(i, g, \lambda) \mathcal{H}$ of $S / \mathcal{H}$ by $(i, \lambda)$. Suppose that the mapping $\phi_{i, j, \mu} \in \Phi$ is defined, so that $\exists \nu \in \Lambda$ such that $(i, \nu) \in X_{\alpha}$, $(j, \mu) \in X_{\beta}$ (for some $\alpha, \beta \in J$ ) and $(j, \mu) \kappa(i, \nu)$. Then in $S^{\prime}=S / \mathcal{H C}=$ $S\left(\Re^{\prime}, \Phi^{\prime}, \Psi^{\prime}\right)$ the structure mapping $\phi_{i, j, \mu}^{\prime} \in \Phi^{\prime}$ is defined: let $(i, \lambda) \phi_{i, j, \mu}^{\prime}=$ $(j, \rho)$ (for some $\rho \in \Lambda$ ). Since the structure mappings commute with $\mathcal{H}^{\dagger}$ (the natural map from $S$ onto $S / \mathcal{F C})$ it follows that $(i, g, \lambda) \phi_{i, j, \mu} \in H_{j \rho}$ : since this is independent of $g$, the mappings in $\Phi$ are $\mathcal{K}$-class preserving. Similarly the mappings in $\Psi$ are $\mathcal{H}$-class preserving.

Suppose conversely that the structure mappings of $S$ are all $\mathcal{H}$-class preserving and take elements $(i, g, \lambda),(i, h, \lambda)$ and $(j, f, \mu)$ in $S$. Let $(k, \nu) \in$ $S(\lambda, j)$. Then by (2),

$$
(i, g, \lambda)(j, f, \mu)=(i, g, \lambda) \psi_{\lambda, \nu, k}(j, f, \mu) \phi_{j, k, \nu}=\left(l, g_{1}, \nu\right)\left(k, f_{1}, \nu\right)
$$

for some $(k, \nu) \in X_{\beta}, \quad l \in I_{\beta}, \nu \in \Lambda_{\beta}, g_{1}, f_{1} \in G_{\beta}$ and $\beta \in J$. Thus $(i, g, \lambda)(j, f, \mu) \in H_{l v}$. Since $\psi_{\lambda, \nu, k}$ is $\mathcal{H}$-class preserving, $(i, h, \lambda) \psi_{\lambda, \nu, k}=$ $\left(l, h_{1}, \nu\right)$ for some $h_{1} \in G_{\beta}$, and it follows again by (2) that $(i, h, \lambda)(j, f, \mu) \in$ $H_{l v}$. Hence $\mathcal{H}$ is a right congruence, and the dual argument shows that $\mathcal{H}$ is a left congruence.

We close this section by relating the above ideas and notation to the notion of a pseudo-inverse semigroup. A pseudo-inverse semigroup was defined by Nambooripad [21] as a regular semigroup whose biordered set is a pseudo- 
semilattice. (A pseudo-semilattice was defined by Schein [24] as a structure $\left(E, \omega^{l}, \omega^{r}\right)$ consisting of a set $E$ together with two quasi-orders $\omega^{l}$ and $\omega^{r}$ on $E$ satisfying the condition that to every pair $e, f \in E$ there corresponds a unique greatest lower bound $e \wedge f$ with respect to $\omega^{l}$ and $\omega^{r}$, that is,

$$
\omega^{l}(e) \cap \omega^{r}(f)=\omega(e \wedge f)
$$

where $\omega=\omega^{l} \cap \omega^{r}$ and $\rho(e)=\{f \in E \mid f \rho e\}$ for $\rho=\omega^{l}, \omega^{r}$ or $\left.\omega.\right)$ In [22], Nambooripad established that the natural partial order on a regular semigroup $S$ is compatible with the multiplication if and only if $S$ is pseudo-inverse. He also established several equivalent characterizations of pseudo-inverse semigroups. We translate one of these equivalent conditions ([22, Theorem 3.3(b)]) into our present notation in the following theorem.

THEOREM 1.8. The following conditions on a regular semigroup $S=$ $S(\mathfrak{N}, \Phi, \Psi)$ are equivalent.

(a) $S$ is pseudo-inverse.

(b) $S$ satisfies the following condition and its dual: if $R_{j}<R_{i}$ for some $i \in I_{\alpha}$, $j \in I_{\beta}(\alpha, \beta \in J)$ and if $(j, \lambda),(j, \mu) \in X_{\beta}$, then $\phi_{i, j, \lambda}=\phi_{i j, \mu} \quad$ (i.e., if $R_{j}<R_{i}$ then there is exactly one structure mapping from $R_{i}$ to $R_{j}$ ).

(c) $S$ satisfies the following condition and its dual: if the structure mappings $\phi_{i, j, \lambda}$ and $\phi_{i, j, \mu}$ are defined, then $\phi_{i, j, \lambda}=\phi_{i, j, \mu}$ (i.e., if $f \kappa e$ then there is exactly one structure mapping from $R_{e}$ to $R_{f}$ ).

Proof. The equivalence of (a) and (b) follows from Proposition 1.1 and Nambooripad's Theorem 3.3 [22]. It is also clear that (c) implies (b), so we need only show that (a) (or (b)) implies (c). Suppose $f, g \kappa e$ and $f \Re g$ : if either $f \omega^{r} e$ or $g \omega^{r} e$, then $\phi_{e, f}=\phi_{e, g}$ by (b), so assume $f \omega^{l} e$ and $g \omega^{l} e$. Since $E(S)$ is a pseudo-semilattice there exists $h \in E(S)$ such that $\omega^{l}(e) \cap$ $\omega^{r}(f)=\omega(h)$. Since $f, g \in \omega^{l}(e) \cap \omega^{r}(f)$ it follows that $f, g \in \omega(h)$. Since $f \Re g$ it follows by (b) that $\phi_{h, f}=\phi_{h, g}$ : hence $f=h \phi_{h, f}=h \phi_{h, g}=g$, i.e., $f=g$. This establishes (c).

2. Structural uniformity. In [14] the author introduced the notion of structural uniformity of the structure mappings of an inverse semigroup. In this section we introduce an analogous notion for regular semigroups.

Let $S=S(\Re, \Phi, \Psi)$ be a regular semigroup. If for some $\alpha, \beta \in J, i \in I_{\alpha}$, $j \in I_{\beta}$ and $\mu \in \Lambda_{\beta}$ the structure mapping $\phi_{i, j, \mu}$ is defined and if $\lambda \in \Lambda_{\alpha}$ then the restriction of $\phi_{i, j, \mu}$ to $H_{i \lambda}$ induces a mapping $\phi_{i, j, \mu}^{\lambda}$ from $G_{\alpha}$ to $G_{\beta}$ as follows: for $g \in G_{\alpha}, g \phi_{i, j, \mu}^{\lambda}=h \in G_{\beta}$ if $(i, g, \lambda) \phi_{i, j, \mu}=(j, h, \nu)$ for some $\nu \in$ $\Lambda_{\beta}$. Similarly a structure mapping $\psi_{\lambda, \mu j}$ (with $\lambda \in \Lambda_{\alpha}, \mu \in \Lambda_{\beta}, j \in I_{\beta}$ ) induces a family of mappings $\psi_{\lambda, \mu, j}^{i}: G_{\alpha} \rightarrow G_{\beta}$ defined for each $i \in I_{\alpha}$ and $g \in G_{\alpha}$ by $g \psi_{\lambda, \mu, j}^{i}=h$ if $(i, g, \lambda) \psi_{\lambda, \mu, j}=(k, h, \mu)$ for some $k \in I_{\beta}$.

Under certain circumstances the mappings $\phi_{i, j, \mu}^{\lambda}$ and $\psi_{\lambda, \mu, j}^{i}$ are group homo- 
morphisms: one condition which guarantees this is provided in the following theorem.

THEOREM 2.1. Suppose that $\mathcal{H}$ is a congruence on the regular semigroup $S=S(\mathfrak{T}, \Phi, \Psi)$. Let $(i, \lambda) \in X_{\alpha}$ and $(j, \mu) \in X_{\beta}$ (for some $\alpha, \beta \in J$ ) and suppose that $(j, \mu) \omega(i, \lambda)$. Then $\phi_{i, j, \mu}^{\lambda}=\psi_{\lambda, \mu j}^{i}$ : if $p_{\lambda i}=1_{\alpha}$ and $p_{\mu j}=1_{\beta}$ then $\phi_{i, j, \mu}^{\lambda}$ is a group homomorphism from $G_{\alpha}$ to $G_{\beta}$.

Proof. Since $\left(j, p_{\mu j}^{-1}, \mu\right) \omega\left(i, p_{\lambda i}^{-1}, \lambda\right)$ it follows that

$$
\left(i, p_{\lambda i}^{-1}, \lambda\right) \phi_{i, j, \mu}=\left(i, p_{\lambda i}^{-1}, \lambda\right) \psi_{\lambda, \mu j}=\left(j, p_{\mu j}^{-1}, \mu\right) .
$$

Thus, since $\mathcal{H}$ is a congruence on $S$, it follows from Corollary 1.7 that $\phi_{i, j, \mu}, \psi_{\lambda, \mu, j}: H_{i \lambda} \rightarrow H_{j \mu}$. Thus for each $g \in G_{\alpha},(i, g, \lambda) \phi_{i, j, \mu}$ and $(i, g, \lambda) \psi_{\lambda, \mu, j}$ are $\mathcal{H}$-related elements of $S$ : but by Proposition $1.1,(i, g, \lambda) \phi_{i, j, \mu}<(i, g, \lambda)$ and $(i, g, \lambda) \psi_{\lambda, \mu, j}<(i, g, \lambda)$. Hence by Nambooripad's Corollary 1.3 [22] it follows that $(i, g, \lambda) \phi_{i, j, \mu}=(i, g, \lambda) \psi_{\lambda, \mu, j}$ and from this it follows that $\phi_{i, j, \mu}^{\lambda}=$ $\psi_{\lambda, \mu, j}^{i}$

Suppose now that $h \in G_{\alpha}$ and consider the product

$$
(i, g, \lambda)(i, h, \lambda)=\left(i, g p_{\lambda i} h, \lambda\right)=(i, g h, \lambda)
$$

since $p_{\lambda i}=1_{\alpha}$. By (k4) of the author's paper [13],

$$
\begin{aligned}
(i, g h, \lambda) \phi_{i, j, \mu} & =[(i, g, \lambda)(i, h, \lambda)] \phi_{i, j, \mu} \\
& =(i, g, \lambda) \psi_{\lambda, \mu, j}(i, h, \lambda) \phi_{i, j, \mu} \\
& =\left(j, g \psi_{\lambda, \mu, j}^{i}, \mu\right)\left(j, h \phi_{i, j, \mu}^{\lambda}, \mu\right) \\
& =\left(j, g \psi_{\lambda, \mu, j}^{i} h \phi_{i, j, \mu}^{\lambda}, \mu\right) \text { since } p_{\mu j}=1_{\beta} .
\end{aligned}
$$

Hence,

$$
(g h) \phi_{i, j, \mu}^{\lambda}=g \psi_{\lambda, \mu, j}^{i} h \phi_{i, j, \mu}^{\lambda}=g \phi_{i, j, \mu}^{\lambda} h \phi_{i, j, \mu}^{\lambda},
$$

and so $\phi_{i, j, \mu}^{\lambda}=\psi_{\lambda, \mu, j}^{i}$ is a group homomorphism.

We now introduce the notion of structural uniformity for regular semigroups.

DEFINITION 2.2. Let $S=S(\mathfrak{T}, \Phi, \Psi)$ be a regular semigroup. The set $\Phi$ of $\Re$-class structure mappings of $S$ is called structurally uniform if $\phi_{i, j, \mu}^{\lambda}=\phi_{i, j, \mu}^{\nu}$ whenever these mappings are defined (i.e., if there exists $\alpha, \beta \in J$ and $\rho \in \Lambda$ such that $\lambda, \nu \in \Lambda_{\alpha},(i, \rho) \in X_{\alpha},(j, \mu) \in X_{\beta}$, and $\left.(j, \mu) \kappa(i, \rho)\right)$. Analogously $\Psi$ is called structurally uniform if $\psi_{\lambda, \mu, j}^{i}=\psi_{\lambda, \mu, j}^{k}$ whenever these mappings are defined (i.e., if there exist $\alpha, \beta \in J$ and $l \in I$ such that $i, k \in I_{\alpha},(l, \lambda) \in I_{\alpha}$, $(j, \mu) \in X_{\beta}$ and $\left.(j, \mu) \kappa(l, \lambda)\right)$. Thus $\Phi$ is structurally uniform if each $\phi_{i, j, \mu}^{\lambda}$ is independent of $\lambda$.

The following theorem relates this idea to a more familiar one. A subset $T$ of $S$ is called a transversal subset of the $\mathcal{H}$-classes of $S$ if $T$ meets each $\mathcal{H}$-class precisely once. 
THEOREM 2.3. The regular semigroup $S$ has a transversal subsemigroup $T$ of its $\mathcal{H}$-classes if and only if $S \cong S(\mathfrak{R}, \Phi, \Psi)$ for some set $\mathfrak{R}=\left\{M_{\alpha} \mid \alpha \in J\right\}$ of Rees groupoids

$$
M_{\alpha}=\mathscr{T}^{0}\left(G_{\alpha} ; I_{\alpha}, \Lambda_{\alpha} ; P_{\alpha}\right) \backslash\left\{0_{\alpha}\right\}
$$

where

(T1) each $P_{\alpha}(\alpha \in J)$ is a $\left(0_{\alpha}, 1_{\alpha}\right)$ matrix over $G_{\alpha}^{0_{\alpha}}$, and

(T2) $\Phi$ and $\Psi$ are structurally uniform.

Proof. Suppose first that $S^{\prime}=S(\mathfrak{T}, \Phi, \Psi)$ satisfies (T1) and (T2). Let

$$
T=\left\{\left(i, 1_{\alpha}, \lambda\right) \mid i \in I_{\alpha}, \lambda \in \Lambda_{\alpha}, \alpha \in J\right\} ;
$$

$T$ is clearly a transversal subset of the $\mathcal{H}$-classes of $S^{\prime}$. Since each $P_{\alpha}$ is a $\left(0_{\alpha}, 1_{\alpha}\right)$ matrix,

$$
E\left(S^{\prime}\right)=\left\{\left(i, 1_{\alpha}, \lambda\right) \mid(i, \lambda) \in X_{\alpha}, \alpha \in J\right\}
$$

and so $E\left(S^{\prime}\right) \subseteq T$. Suppose that $(i, \lambda) \in X_{\alpha},(j, \mu) \in X_{\beta}$ and $(j, \mu) \kappa(i, \lambda)$. Then by condition (k2) of [13], $\exists \nu \in \Lambda_{\beta}, k \in I_{\beta}$ such that

$$
\left(i, 1_{\alpha}, \lambda\right) \phi_{i, j, \mu}=\left(j, 1_{\beta}, \nu\right) \text { and }\left(i, 1_{\alpha}, \lambda\right) \psi_{\lambda, \mu j}=\left(k, 1_{\beta}, \mu\right) \text {. }
$$

It follows by (T2) that if $\rho \in \Lambda_{\alpha}, \exists \tau \in \Lambda_{\beta}$ such that

$$
\left(i, 1_{\alpha}, \rho\right) \phi_{i, j, \mu}=\left(j, 1_{\beta}, \tau\right) \text {, }
$$

and if $l \in I_{\alpha}, \exists m \in I_{\beta}$ such that

$$
\left(l, 1_{\alpha}, \lambda\right) \psi_{\lambda, \mu, j}=\left(m, 1_{\beta}, \mu\right) .
$$

Now let $\left(i, 1_{\alpha}, \lambda\right)$ and $\left(j, 1_{\beta}, \mu\right)$ be any two elements of $T$ and let $\left(k, 1_{\gamma}, \nu\right) \in$ $S(\lambda, j)$. Then by (2),

$$
\begin{aligned}
\left(i, 1_{\alpha}, \lambda\right)\left(j, 1_{\beta}, \mu\right) & =\left(i, 1_{\alpha}, \lambda\right) \psi_{\lambda, \nu, k}\left(j, 1_{\beta}, \mu\right) \phi_{j, k, \nu} \\
& =\left(l, 1_{\gamma}, \nu\right)\left(k, 1_{\gamma}, \rho\right), \text { for some } l \in I_{\gamma}, \rho \in \Lambda_{\gamma} \\
& =\left(l, 1_{\gamma}, \rho\right) \in T \text { since } p_{\nu k}=1_{\gamma} .
\end{aligned}
$$

Hence $T$ is a transversal subsemigroup of the $\mathcal{H}$-classes of $S^{\prime}$. The isomorphism $S \simeq S^{\prime}$ provides a transversal subsemigroup of the $\mathcal{H}$-classes of $S$.

Suppose, conversely, that $S$ has a transversal subsemigroup $T$ of its $\mathcal{C}$-classes. For each $\mathscr{D}$-class $D_{\alpha}$ of $S, D_{\alpha}^{0_{\alpha}}=D_{\alpha} \cup\left\{0_{\alpha}\right\}$ is a completely 0 -simple semigroup which has a transversal subsemigroup of its $\mathcal{H}$-classes, so by Théorème 4.13 and Corollaire 4.10 of Lallement and Petrich [10], $D_{\alpha}^{0_{\alpha}} \simeq$ $\mathfrak{T}^{0}\left(G_{\alpha} ; I_{\alpha}, \Lambda_{\alpha} ; P_{\alpha}\right)$ for some $\left(0_{\alpha}, 1_{\alpha}\right)$ matrix $P_{\alpha}$. Hence we may assume without loss of generality that $S=S(\mathfrak{R}, \Phi, \Psi)$ where $\mathfrak{N}=\left\{M_{\alpha} \mid \alpha \in J\right\}$,

$$
M_{\alpha}=\Re^{0}\left(G_{\alpha} ; I_{\alpha}, \Lambda_{\alpha} ; P_{\alpha}\right) \backslash\left\{0_{\alpha}\right\}
$$

and each $P_{\alpha}$ is a $\left(0_{\alpha}, 1_{\alpha}\right)$ matrix over $G_{\alpha}^{0_{\alpha}}$. Thus

$$
E(S)=\left\{\left(i, 1_{\alpha}, \lambda\right) \mid(i, \lambda) \in X_{\alpha}, \alpha \in J\right\}
$$


Let $T=\left\{\left(i, g_{i \lambda}, \lambda\right) \mid i \in I_{\alpha}, \lambda \in \Lambda_{\alpha}, \alpha \in J\right\}$; the group elements $g_{i \lambda}$ satisfy the following properties:

(3) if $(j, \lambda) \in X_{\alpha}, i \in I_{\alpha}$ and $\mu \in \Lambda_{\alpha}$ then $g_{i \lambda} g_{j \mu}=g_{i \mu}$;

(4) if $(i, \lambda) \in X_{\alpha}$ then $g_{i \lambda}=1_{\alpha}$, and so $E(S) \subseteq T$;

(5) if $(j, \lambda),(i, \mu) \in X_{\alpha}$ then $g_{i \lambda}=g_{j \mu}^{-1}$;

(6) if $i \in I_{\alpha}, \lambda \in \Lambda_{\alpha}$ and $\phi_{i, j, \mu} \in \Phi$, then

$$
\left(i, g_{i \lambda}, \lambda\right) \phi_{i j, \mu}=\left(j, g_{j p}, \nu\right) \text { for some } \nu \in \Lambda \text {; }
$$

(7) if $i \in I_{\alpha}, \lambda \in \Lambda_{\alpha}$ and $\psi_{\lambda, \mu, j} \in \Psi$, then

$$
\left(i, g_{i \lambda}, \lambda\right) \psi_{\lambda, \mu j}=\left(k, g_{k \mu}, \mu\right) \text { for some } k \in I \text {. }
$$

Property (3) is verified by noting that $\left(i, g_{i \lambda}, \lambda\right)\left(j, g_{j \mu}, \mu\right)=\left(i, g_{i \mu}, \mu\right)$ and (4) and (5) may be verified in a similar way. Property (6) is verified by noticing that

$$
\left(i, g_{i \lambda}, \lambda\right) \phi_{i, j, \mu}=\left(j, 1_{\beta}, \mu\right)\left(i, g_{i \lambda}, \lambda\right)
$$

(where $\left.j \in I_{\beta}\right)$ and so $\left(i, g_{i \lambda}, \lambda\right) \phi_{i, j, \mu} \in T$ since $\left(j, 1_{\beta}, \mu\right) \in T$ by (4) and $\left(i, g_{i \lambda}, \lambda\right) \in T$; a similar argument shows that (7) is satisfied.

For each $\alpha \in J$ choose (and fix) elements $i(\alpha) \in I_{\alpha}$ and $\lambda(\alpha) \in \Lambda_{\alpha}$ such that $(i(\alpha), \lambda(\alpha)) \in X_{\alpha}$. For each $i \in I_{\alpha}$ (and each $\left.\alpha \in J\right)$ choose (and fix) an element $\lambda(i) \in \Lambda_{\alpha}$ such that $(i, \lambda(i)) \in X_{\alpha}$ : for each $\lambda \in \Lambda_{\alpha}$ choose (and fix) an element $i(\lambda) \in I_{\alpha}$ such that $(i(\lambda), \lambda) \in X_{\alpha}$. Define an idempotent-separating bijection $\theta: S \rightarrow S$ by

$$
\theta:(i, g, \lambda) \mapsto\left(i, g_{i(\alpha), \lambda(i)} g g_{i(\lambda), \lambda(\alpha)}, \lambda\right)
$$

$\forall i \in I_{\alpha}, g \in G_{\alpha}, \lambda \in \Lambda_{\alpha}$ and $\alpha \in J$.

We claim first that $\theta$ is a (partial groupoid) homomorphism from $\operatorname{tr}(S)$ onto $\operatorname{tr}(S)$. Let $(j, \lambda) \in X_{\alpha}, i \in I_{\alpha}, \mu \in \Lambda_{\alpha}$ and $g, h \in G_{\alpha}$ for some $\alpha \in J$, so that $(i, g, \lambda)(j, h, \mu)=(i, g h, \mu)$ is a product in $\operatorname{tr}(S)$. Then

$$
\begin{aligned}
(i, g, \lambda) \theta(j, h, \mu) \theta & =\left(i, g_{i(\alpha), \lambda(i)} g g_{i(\lambda), \lambda(\alpha)}, \lambda\right)\left(j, g_{i(\alpha), \lambda(j)} h g_{i(\mu), \lambda(\alpha)}, \mu\right) \\
& =\left(i, g_{i(\alpha), \lambda(i)} g g_{i(\lambda), \lambda(\alpha)} g_{i(\alpha), \lambda(j)} h g_{i(\mu), \lambda(\alpha)}, \mu\right) \\
& =\left(i, g_{i(\alpha), \lambda(i)} g g_{i(\lambda), \lambda(j)} h g_{i(\mu), \lambda(\alpha)}, \mu\right) \text { by (3). }
\end{aligned}
$$

Now $(i(\lambda), \lambda) \in X_{\alpha},(j, \lambda(j)) \in X_{\alpha}$ and $(j, \lambda) \in X_{\alpha}$, so, by (4), (i( $\left.\left.\lambda\right), 1_{\alpha}, \lambda\right)$, $\left(j, 1_{\alpha}, \lambda(j)\right) \in T$ and so

$$
\left(i(\lambda), 1_{\alpha}, \lambda\right)\left(j, 1_{\alpha}, \lambda(j)\right)=\left(i(\lambda), 1_{\alpha}, \lambda(j)\right) \in T .
$$

Hence $g_{i(\lambda), \lambda(j)}=1_{\alpha}$ and it follows that

$$
\begin{aligned}
(i, g, \lambda) \theta(j, h, \mu) \theta & =\left(i, g_{i(\alpha), \lambda(i)} g h g_{i(\mu), \lambda(\alpha)}, \mu\right) \\
& =(i, g h, \mu) \theta=[(i, g, \lambda)(j, h, \mu)] \theta .
\end{aligned}
$$

Hence $\theta$ is a trace homomorphism from $\operatorname{tr}(S)$ onto $\operatorname{tr}(S)$.

We claim next that $\theta^{-1}$ is also a trace homomorphism from $\operatorname{tr}(S)$ onto $\operatorname{tr}(S)$, and so $\theta$ is a trace isomorphism from $\operatorname{tr}(S)$ onto $\operatorname{tr}(S)$. Again let 
$(j, \lambda) \in X_{\alpha}, i \in I_{\alpha}, \mu \in \Lambda_{\alpha}$ and $g, h \in G_{\alpha}$, so that

$$
(i, g, \lambda)(j, h, \mu)=(i, g h, \mu)
$$

is a product in $\operatorname{tr}(S)$. Then

$$
\begin{aligned}
(i, g, \lambda) \theta^{-1}(j, h, \mu) \theta^{-1} & =\left(i, g_{i(\alpha), \lambda(i)}^{-1} g g_{i(\lambda), \lambda(\alpha)}^{-1}, \lambda\right)\left(j, g_{i(\alpha), \lambda(j)}^{-1} h g_{i(\mu), \lambda(\alpha)}^{-1}, \mu\right) \\
& =\left(i, g_{i(\alpha), \lambda(i)}^{-1} g g_{i(\lambda), \lambda(\alpha)}^{-1} g_{i(\alpha), \lambda(j)}^{-1} h g_{i(\mu), \lambda(\alpha)}^{-1}, \mu\right) \\
& =\left(i, g_{i(\alpha), \lambda(i)}^{-1} g g_{i(\alpha), \lambda} g_{j, \lambda(\alpha)} h g_{i(\mu), \lambda(\alpha)}^{-1}, \mu\right) \text { by (5) } \\
& =\left(i, g_{i(\alpha), \lambda(i)}^{-1} g g_{i(\alpha), \lambda(\alpha)} h g_{i(\mu), \lambda(\alpha)}^{-1}, \mu\right) \text { by (3) } \\
& =\left(i, g_{i(\alpha), \lambda(i)}^{-1} g h g_{i(\mu), \lambda(\alpha)}^{-1}, \mu\right) \text { by (4) } \\
& =(i, g h, \mu) \theta^{-1}=[(i, g, \lambda)(j, h, \mu)] \theta^{-1}
\end{aligned}
$$

Hence $\theta^{-1}$ is a trace isomorphism from $\operatorname{tr}(S)$ onto $\operatorname{tr}(S)$. Now if $\theta$ is a partial groupoid isomorphism from the $\operatorname{trace} \operatorname{tr}(S)$ of a regular semigroup $S=$ $S(\Re, \Phi, \Psi)$ onto any partial groupoid $S^{\prime}$, then $\theta$ induces a multiplication defined by $(a \theta) \cdot(b \theta)=(a b) \theta$ on $S^{\prime}$ in such a way that $\left(S^{\prime}, \cdot\right)$ becomes a regular semigroup with trace $S^{\prime}$ and set $\Phi^{\prime} \cup \Psi^{\prime}$ of structure mappings, where

$$
\phi_{i, j, \lambda}^{\prime}=\theta^{-1} \phi_{i, j, \lambda} \theta \quad \forall \phi_{i, j, \lambda} \in \Phi,
$$

and

$$
\psi_{\lambda, \mu, j}^{\prime}=\theta^{-1} \psi_{\lambda, \mu, j} \theta \quad \forall \psi_{\lambda, \mu, j} \in \Psi
$$

Furthermore, $\theta$ is an isomorphism from $S$ onto $S^{\prime}$.

So define a new set $\Phi^{\prime} \cup \Psi^{\prime}$ of structure mappings on $S$ by (8) and (9), and denote the new semigroup $S\left(\mathfrak{T}, \Phi^{\prime}, \Psi^{\prime}\right)$ by $S^{\prime}$. We claim that $\Phi^{\prime}$ and $\Psi^{\prime}$ are structurally uniform. To see this we first suppose that $\phi_{i, j, \mu}$ is defined for some $i \in I_{\alpha},(j, \mu) \in X_{\beta}$ and that $\lambda \in \Lambda_{\alpha}$. Then

$$
\begin{aligned}
& \left(i, 1_{\alpha}, \lambda\right) \phi_{i, j, \mu}^{\prime}=\left(i, 1_{\alpha}, \lambda\right) \theta^{-1} \phi_{i, j, \mu} \theta \\
& =\left(i, g_{i(\alpha), \lambda(i)}^{-1} g_{i(\lambda), \lambda(\alpha)}^{-1}, \lambda\right) \phi_{i, j, \mu} \theta \\
& =\left(i, g_{i, \lambda(\alpha)} g_{i(\alpha), \lambda}, \lambda\right) \phi_{i, j, \mu} \theta \text { by (5) } \\
& =\left(i, g_{i \lambda}, \lambda\right) \phi_{i, j, \mu} \theta \text { by (3) } \\
& =\left(j, g_{j, \nu}, \nu\right) \theta, \quad \text { for some } \nu \in \Lambda_{\beta} \text {, by (6) } \\
& =\left(j, g_{i(\beta), \lambda(j)} g_{j, \nu} g_{i(\nu), \lambda(\beta)}, \nu\right) \\
& =\left(j, g_{i(\beta), \nu} g_{i(\nu), \lambda(\beta)}, \nu\right) \text { by (3) } \\
& =\left(j, g_{i(\beta), \lambda(\beta)}, \nu\right) \text { by (3) } \\
& =\left(j, 1_{\beta}, \nu\right) \text { by (4). }
\end{aligned}
$$


A similar argument shows that if $\psi_{\lambda, \mu_{j}}$ is defined for some $\lambda \in \Lambda_{\alpha},(j, \mu) \in$ $X_{\beta}$ and if $i \in I_{\alpha}$, then there exists $k \in I_{\beta}$ such that $\left(i, 1_{\alpha}, \lambda\right) \psi_{\lambda, \mu_{j}}^{\prime}=\left(k, 1_{\beta}, \mu\right)$. Suppose now that $(i, \lambda) \in X_{\alpha}, \mu \in \Lambda_{\alpha},(j, \nu) \in X_{\beta},(j, \nu) \kappa(i, \lambda)$ and $(i, g, \lambda) \phi_{i, j, \nu}^{\prime}=(j, h, \rho)$ for some $\rho \in \Lambda_{\beta}$. We aim to show that $(i, g, \mu) \phi_{i, j, \nu}^{\prime}=$ $(j, h, \tau)$ for some $\tau \in \Lambda_{\beta}$. Let $a=(i, g, \lambda), a^{\prime}=\left(i, g^{-1}, \lambda\right)$ (the inverse of $a$ in $\left.H_{i \lambda}\right), e=a a^{\prime}=a^{\prime} a=\left(i, 1_{\alpha}, \lambda\right), f=\left(j, 1_{\beta}, \nu\right)$ and $b=(i, g, \mu)$. Then $f \kappa e$ in $E\left(S^{\prime}\right)$ and $a^{\prime} f a \omega e$ in $E\left(S^{\prime}\right)$. Let $a^{\prime} f a=\left(k, 1_{\beta}, \rho\right)$, some $k \in I_{\beta}$, so that $(k, \rho) \in X_{\beta}$. Then

$$
\begin{aligned}
(i, g, \mu) \phi_{i, j, \nu}^{\prime} & =b \phi_{e, f}^{\prime}=f b=(f a)\left[\left(a^{\prime} f a\right)\left(a^{\prime} b\right)\right] \\
& =\left(a \phi_{e, f}^{\prime}\right)\left[\left(a^{\prime} b\right) \phi_{e, a^{\prime} f a}^{\prime}\right] \\
& =(j, h, \rho)\left[\left(i, g^{-1}, \lambda\right)(i, g, \mu)\right] \phi_{i, k, \rho}^{\prime} \\
& =(j, h, \rho)\left[\left(i, 1_{\alpha}, \mu\right) \phi_{i, k, \rho}^{\prime}\right] \quad \text { since }(i, \lambda) \in X_{\alpha} \\
& =(j, h, \rho)\left(k, 1_{\beta}, \tau\right), \quad \text { for some } \tau \in \Lambda_{\beta} \\
& =(j, h, \tau) \quad \text { since }(k, \rho) \in X_{\beta} .
\end{aligned}
$$

Hence $\Phi^{\prime}$ is structurally uniform: a similar argument shows that $\Psi^{\prime}$ is structurally uniform. This completes the proof of the theorem.

3. The fundamental four-spiral semigroup. In [1], K. Byleen, F. Pastijn and the present author introduced a semigroup $\mathrm{Sp}_{4}$ which is a basic building block for bisimple idempotent-generated semigroups. $\mathrm{Sp}_{4}$ is a fundamental bisimple idempotent-generated semigroup whose biordered set of idempotents is isomorphic to the four-spiral $E_{4}=\left\{a_{n}, b_{n}, c_{n}, d_{n} \mid n \in N\right\}$ described in [1] and pictured in Diagram 1 below.

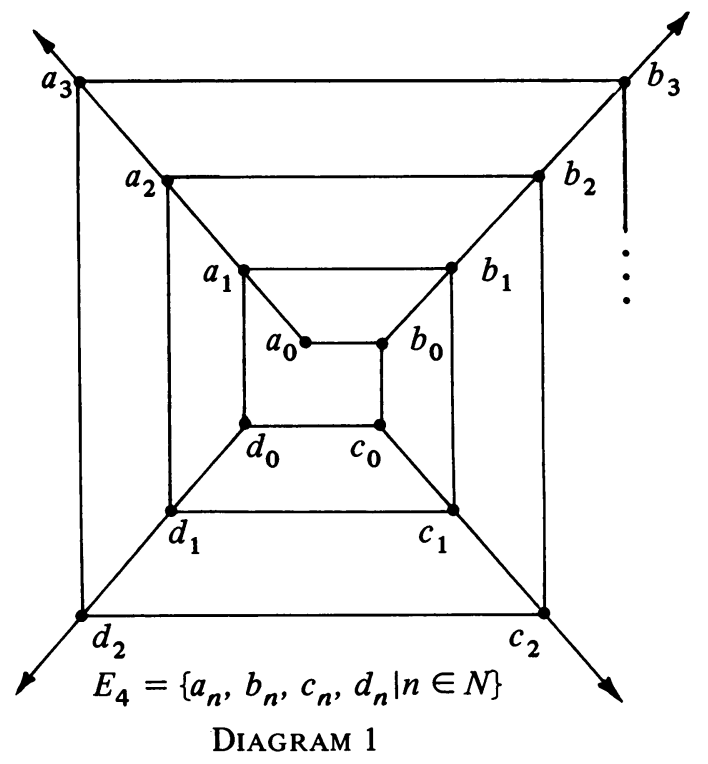


In this diagram, elements in the same row are $\mathcal{R}$-related, elements in the same column are $\mathcal{L}$-related, and the natural order proceeds down the four diagonals away from the centre. The semigroup $\mathrm{Sp}_{4}$ is isomorphic to the semigroup $\bar{T}_{E_{4}}$ (in the sense of Nambooripad [19]) and is the only fundamental regular semigroup whose biordered set is (biorder isomorphic to) $E_{4}$. The structure of $\mathrm{Sp}_{4}$ was described in several different ways in [1]. In this section we describe $\mathrm{Sp}_{4}$ as a semigroup of ordered pairs of integers: this form of the multiplication for $\mathrm{Sp}_{4}$ will be most useful when describing the $\mathcal{H}$-coextensions of $\mathrm{Sp}_{4}$.

We begin by writing down an explicit formula for the sandwich set $S(e, f)$ of two idempotents $e$ and $f$ in $E_{4}$. Recall from [1] that by $B_{4}$ we mean the four-element rectangular band $B_{4}=\{a, b, c, d\}$ pictured in Diagram 2.

\begin{tabular}{|l|l|}
\hline$a$ & $b$ \\
\hline$d$ & $c$ \\
\hline
\end{tabular}

$$
B_{4}=\{a, b, c, d\}
$$

DiAgRAM 2

We let $N$ denote the set $N=\{0,1,2,3, \ldots\}$ of natural numbers, let $Z$ denote the set of integers, and use the notation $E_{4}=\left\{u_{n} \mid n \in N, u \in B_{4}\right\}$ for the elements of the four-spiral biordered set $E_{4}$. For $n, m \in Z$ we denote $\max \{m, n\}$ by $m \vee n$. It is routine to verify the following proposition.

Proposition 3.1. If $u_{n}, v_{m} \in E_{4}$, then $S\left(u_{n}, v_{m}\right)=\left\{(v u)_{p}\right\}$ where vu denotes the product of $v$ and $u$ in $B_{4}$ and

$$
p= \begin{cases}(n-1) \vee m & \text { if } u=a \text { and } v \in\{c, d\}, \\ (n+1) \vee m & \text { if } u=d \text { and } v \in\{a, b\}, \\ n \vee m & \text { otherwise. }\end{cases}
$$

The elements of $\mathrm{Sp}_{4}=\bar{T}_{E_{4}}$ may be described as equivalence classes of isomorphisms between principal ideals of $E_{4}$ modulo the equivalence relation $\sigma$ introduced by Nambooripad [19] in his description of $\bar{T}_{E}$. In [1] we denoted the $\sigma$-equivalence class of the principal ideal isomorphism which carries $u_{n}$ onto $v_{m}$ by $\left(u_{n}, v_{m}\right)$. From Nambooripad's definition of $\sigma$ it follows that

$$
\left(u_{n}, v_{m}\right)=\left(w_{k}, x_{l}\right) \text { iff } u_{n} \Re w_{k} \text { and } v_{m} \mathcal{E} x_{l} \text {. }
$$

Thus the distinct elements of $\mathrm{Sp}_{4}$ are as follows:

$$
\mathrm{Sp}_{4}=\left\{\left(u_{n}, u_{m}\right) \mid m, n \in N, u \in B_{4}\right\} \cup\left\{\left(d_{n}, a_{0}\right) \mid n \in N\right\} \text {. }
$$

We denote the idempotent $\left(u_{n}, u_{n}\right)\left(u \in B_{4}, n \in N\right)$ simply by $u_{n}$ and identify it with the corresponding element of $E_{4}$ : thus $\left(u_{n}, v_{m}\right) \in R_{u_{n}} \cap L_{v_{m}}$. Multiplication in $\mathrm{Sp}_{4}$ is by means of equation (1) (\$1). Let $\left(u_{n}, v_{m}\right),\left(w_{k}, x_{l}\right) \in S$ 
and let $\left(y_{p}, z_{q}\right) \in S\left(v_{m}, w_{k}\right):$ then

$$
\left(u_{n}, v_{m}\right)\left(w_{k}, x_{l}\right)=\left(u_{n}, v_{m}\right) \psi_{v_{m}, z_{q}}\left(w_{k}, x_{l}\right) \phi_{w_{k} y_{p}} \cdot
$$

The structure mappings $\psi_{v_{m}, z_{q}}: L_{v_{m}} \rightarrow L_{z_{q}}$ for $z_{q} \omega^{l} v_{m}$ and $\phi_{w_{k} y_{p}}: R_{w_{k}} \rightarrow R_{y_{p}}$ for $y_{p} \omega^{r} w_{k}$ were discussed in [1]: they move elements of $\mathrm{Sp}_{4}$ down the diagonals in Diagram 4 of [1]. We now translate this into a notation which is more appropriate for the $\mathcal{H}$-coextension discussed in the following section.

Define a mapping $\theta: \mathrm{Sp}_{4} \rightarrow Z \times Z$ as follows: for $m, n \in N$,

$$
\begin{gathered}
\left(a_{m}, a_{n}\right) \theta=(m, n), \quad\left(b_{m}, b_{n}\right) \theta=(m,-(n+1)), \\
\left(c_{m}, c_{n}\right) \theta=(-(m+1),-(n+1)), \quad\left(d_{m}, d_{n}\right) \theta=(-(m+1), n+1),
\end{gathered}
$$

and

$$
\left(d_{m}, a_{0}\right) \theta=(-(m+1), 0) .
$$

The mapping $\theta$ is clearly a bijection from $\operatorname{Sp}_{4}$ onto $Z \times Z$ : if $\left(u_{m}, v_{n}\right) \theta=$ $(i, j)$ then we denote $L_{v_{n}}$ by $L_{j}$ and $R_{u_{m}}$ by $R_{i}$, so that $(i, j) \theta^{-1} \in R_{i} \cap L_{j}$. If $w_{k} \in L_{j}$ and $x_{l} \in R_{i}$ we shall denote the sandwich set $S\left(w_{k}, x_{l}\right)$ by $S(j, i)$. We reformulate Proposition 3.1 in this new notation: if $S(j, i)=\left\{u_{m}\right\}$ and $\left(u_{m}, u_{m}\right) \theta=(k, l)$ we simply write $S(j, i)=(k, l)$.

Proposition 3.2. For $n, r \in Z$ we have

$$
S(n, r)= \begin{cases}(n \vee r, n \vee r) & \text { if } n>0 \text { and } r>0, \\ (-(n \vee-r), n \vee-r) & \text { if } n>0 \text { and } r<0, \\ ((-(n+1)) \vee r,-(-n \vee r+1)) & \text { if } n<0 \text { and } r \geqslant 0 \\ (-(-n \vee-r),-(-n \vee-r)) & \text { if } n<0 \text { and } r<0\end{cases}
$$

Proof. We provide a verification of this in case $n<0$ and $r>0$ : the other cases are similar. If $n<0$ and $r \geqslant 0$ then

$$
\begin{aligned}
S(n, r) & =S\left(b_{-(n+1)}, a_{r}\right)=\left\{b_{(-(n+1)) \vee r}\right\} \\
& =((-(n+1)) \vee r,-(((-(n+1)) \vee r)+1)) \\
& =((-(n+1)) \vee r,-(-n \vee(r+1))),
\end{aligned}
$$

as required. We denote the structure mapping from $R_{i}$ to $R_{j}$ for $0<i<j$ or $0>i>j$ by $\phi_{i, j}$. Similarly the structure mapping from $L_{i}$ to $L_{j}$ for $0<i<j$ or $0>i>j$ is denoted by $\psi_{i, j}$. We translate the description of these structure mappings given in [1] into the present notation. For each $n \in Z$ we define $\sigma(n)$ by

$$
\sigma(n)= \begin{cases}+1 & \text { if } n \geqslant 0 \\ -1 & \text { if } n<0\end{cases}
$$

Then for each $(m, n) \in Z \times Z$ with $R_{m}>R_{p}$ and $L_{n}>L_{q}$, we have

$$
(m, n) \theta^{-1} \phi_{m p}=(p, n+\sigma(n)|p-m|) \theta^{-1} \text {, }
$$


and

$$
(m, n) \theta^{-1} \psi_{n, q}=(m+\sigma(m)|q-n|, q) \theta^{-1} .
$$

Equation (11) and the multiplication (10) enable us to define a multiplication * on $Z \times Z$ in such a way that $(Z \times Z, *) \cong \mathrm{Sp}_{4}$. We summarize this discussion in the following proposition; the multiplication $*$ is of course defined in such a way that $\theta$ becomes an isomorphism from $\mathrm{Sp}_{4}$ onto $(Z \times Z, *)$

Propos ${ }_{\text {lo }}$ ION 3.3. Define a multiplication * on $Z \times Z$ by

$$
(m, n) *(r, s)=(m+\sigma(m)|q-n|, s+\sigma(s)|p-r|)
$$

where $(p, q)=S(n, r)$ is determined by Proposition 3.2. Then $(Z \times Z, *)$ is a semigroup isomorphic (by means of $\theta^{-1}$ ) to $\mathrm{Sp}_{4}$.

We identify $\mathrm{Sp}_{4}$ with $(Z \times Z, *)$ in the sequel and regard $\mathrm{Sp}_{4}$ as a semigroup of pairs of integers. The following result follows easily from the results of Nambooripad [21]. It was also known to Nambooripad (private communication).

Proposition 3.4. The semigroup $\mathrm{Sp}_{4}$ is pseudo-inverse.

Proof. This follows immediately from Nambooripad's Theorem 2.5 [21] since in $E_{4}$ sandwich sets are trivial: thus $E_{4}$ is a pseudo-semilattice and thus $\mathrm{Sp}_{4}$ is pseudo-inverse. In fact it follows that $E_{4}$ is also partially associative in the sense of Nambooripad [21].

We shall close this section with a technical proposition which will be essential for the proof of associativity in Lemma 4.2 of the next section. We first re-state a result due to Nambooripad [18] which we shall need.

Lemma 3.5 (NAmbooripad [18, Lemma 3.9] or [21, Lemma 1.4]). Let $\left(E, \omega^{r}, \omega^{l}, \tau\right)$ be a biordered set, $\alpha: \omega(e) \rightarrow \omega(f)$ an $\omega$-isomorphism and $g, d \in$ $E$. Let $h_{1} \in S(g, e), h_{2} \in S(f, d)$,

$$
h_{1}^{\prime}=\left(h_{1} \wedge e\right) \alpha, \quad h_{2}^{\prime}=\left(f \wedge h_{2}\right) \alpha^{-1} .
$$

Then there exist $h \in S\left(g, h_{2}^{\prime}\right)$ and $h^{\prime} \in S\left(h_{1}^{\prime}, d\right)$ such that $(h \wedge e) \alpha=f \wedge h^{\prime}$. (Here $e \wedge f$ is defined by

$$
e \wedge f= \begin{cases}e & \text { if } e \omega^{r} f, \\ e \tau^{l}(f) & \text { if } e \omega^{l} f, \\ f \tau^{r}(e) & \text { if } f \omega^{r} e, \\ f & \text { if } f \omega^{l} e,\end{cases}
$$

and $e \wedge f$ is undefined otherwise.) 
Proposition 3.6. Let $(m, n),(r, s),(t, u) \in \mathrm{Sp}_{4}$, let $(p, q)=S(n, r)$,

$$
(i, j)=S(s, t), \quad(v, w)=S(s+\sigma(s)|p-r|, t)
$$

and $(k, l)=S(n, r+\sigma(r)|j-s|)$. Then

$$
|p-r|+|w-s-\sigma(s)| p-r||=|j-s|+|k-r-\sigma(r)| j-s|| \text {. }
$$

Proof. This may of course be proved by exhaustion (i.e., by checking cases, using Proposition 3.2). We choose instead to use Nambooripad's lemma (Lemma 3.5). For the purpose of this proposition only we fix idempotents in $L_{n}, R_{r}, L_{s}$ and $R_{t}$ and denote them by $e_{n}, e_{r}, e_{s}$ and $e_{t}$, respectively, and let $\alpha: \omega\left(e_{r}\right) \rightarrow \omega\left(e_{s}\right)$ denote the principal ideal isomorphism from the $\omega$-chain $\omega\left(e_{r}\right)$ onto the $\omega$-chain $\omega\left(e_{s}\right)$. If $z_{1}, z_{2} \in \omega\left(e_{r}\right)$ then by $l\left(z_{1}, z_{2}\right)$ we mean the distance between $z_{1}$ and $z_{2}$ in the $\omega$-chain $\omega\left(e_{r}\right)$ : then

$$
l\left(z_{1}, z_{2}\right)=l\left(z_{1} \alpha, z_{2} \alpha\right) \quad \forall z_{1}, z_{2} \in \omega\left(e_{r}\right)
$$

and if $z_{1} \omega z_{2} \omega e_{r}$ then $l\left(z_{1}, e_{r}\right)=l\left(z_{1}, z_{2}\right)+l\left(z_{2}, e_{r}\right)$. We now apply Nambooripad's Lemma 3.5 with the elements $g, e, f, d$ in the statement of the lemma replaced by $e_{n}, e_{r}, e_{s}$ and $e_{t}$, respectively. We then have

$$
\begin{array}{cc}
h_{1}=S(g, e)=(p, q), & h_{1} \wedge e \in R_{p}, \\
h_{2}=S(f, d)=(i, j), & f \wedge h_{2} \in L_{j} .
\end{array}
$$

Now $h_{1} \wedge e \in \omega(e)$ and $l\left(h_{1} \wedge e, e\right)=|p-r|$, so

$$
l\left(\left(h_{1} \wedge e\right) \alpha, f\right)=|p-r|, \quad \text { so } h_{1}^{\prime}=\left(h_{1} \wedge e\right) \alpha \in L_{s+o(s)|p-r|} .
$$

A similar argument shows that

$$
h_{2}^{\prime}=\left(f \wedge h_{2}\right) \alpha^{-1} \in R_{r+o(r)|j-s|}
$$

Then

$$
\begin{gathered}
h=S\left(g, h_{2}^{\prime}\right)=S(n, r+\sigma(r)|j-s|)=(k, l), \quad h \wedge e \in R_{k}, \\
h^{\prime}=S\left(h_{1}^{\prime}, d\right)=S(s+\sigma(s)|p-r|, t)=(v, w)
\end{gathered}
$$

and $f \wedge h^{\prime} \in L_{w}$. By Lemma 3.5, $(h \wedge e) \alpha=f \wedge h^{\prime}$ and so

$$
l(h \wedge e, e)=l\left(f \wedge h^{\prime}, f\right) \text {. }
$$

But $(h \wedge e) \omega h_{2}^{\prime} \omega e$, so

$$
\begin{aligned}
l(h \wedge e, e) & =l\left(h \wedge e, h_{2}^{\prime}\right)+l\left(h_{2}^{\prime}, e\right) \\
& =|k-r-\sigma(r)| j-s||+|r-r-\sigma(r)| j-s|| \\
& =|j-s|+|k-r-\sigma(r)| j-s|| .
\end{aligned}
$$

Similarly

$$
l\left(f \wedge h^{\prime}, f\right)=|p-r|+|w-s-\sigma(s)| p-r||,
$$

so the result follows. 
4. The general regular four-spiral semigroup. A semigroup $S$ is called a four-spiral semigroup if $E(S)$ is (biorder isomorphic to) the four-spiral biordered set $E_{4}$. In this section we determine the structure of all such regular semigroups $S$ (such a semigroup is clearly bisimple since $E_{4}$ is connected in the sense of [1]).

TheOREM 4.1. Let $G$ be a group and $\alpha$ an endomorphism of $G$. Define $a$ multiplication - on $Z \times G \times Z$ for $m, n, r, s \in Z$ and $g, h \in G$ by

$$
(m, g, n) \cdot(r, h, s)=\left(m+\sigma(m)|q-n|, g \alpha^{|q-n|} h \alpha^{|p-r|}, s+\sigma(s)|p-r|\right)
$$

where $(p, q)=S(n, r)$ and where $\alpha^{0}$ is interpreted as the identity automorphism of $G$. Then $(Z \times G \times Z, \cdot)$ is a regular four-spiral semigroup.

We break the proof into two lemmas.

LEMma 4.2. The multiplication - defined by (13) is associative.

Proof. Let $(m, g, n),(r, h, s),(t, f, u) \in Z \times G \times Z$ : identify $(m, g, n)$ with the pair $[g,(m, n)] \in G \times(Z \times Z)$. Then

$$
(m, g, n) \cdot(r, h, s)=\left(g \alpha^{|q-n|} h \alpha^{|p-r|},(m, n) *(r, s)\right),
$$

and since * is associative we need only verify the following: if $(p, q)=$ $S(n, r)$,

$$
(v, w)=S(s+\sigma(s)|p-r|, t),(i, j)=S(s, t)
$$

and

$$
(k, l)=S(n, r+\sigma(r)|j-s|)
$$

then

$$
\begin{aligned}
g \alpha^{|q-n|+|w-s-\sigma(s)| p-r||} h \alpha^{|p-r|+|w-s-\sigma(s)| p-r||} f \alpha^{|v-t|} \\
=g \alpha^{|l-n|} h \alpha^{|j-s|+|k-r-\sigma(r)| j-s \mid} f \alpha^{|i-t|+|k-r-\sigma(r)| j-s||} .
\end{aligned}
$$

Thus we must verify the following three identities:

$$
\begin{gathered}
|q-n|+|w-s-\sigma(s)| p-r||=|l-n| \\
|p-r|+|w-s-\sigma(s)| p-r||=|j-s|+|k-r-\sigma(r)| j-s|| \\
|v-t|=|i-t|+|k-r-\sigma(r)| j-s||
\end{gathered}
$$

We use associativity of the multiplication * . By comparing first components of $[(m, n) *(r, s)] *(t, u)$ and $(m, n) *[(r, s) *(t, u)]$ we see that

$$
\begin{gathered}
m+\sigma(m)|q-n|+\sigma(m+\sigma(m)|q-n|)|w-s-\sigma(s)| p-r|| \\
=m+\sigma(m)|l-n| .
\end{gathered}
$$

Now $p, q, w$ and $l$ depend only on $n, r, s$ and $t$ and none of these depends on $m$, so we may choose $m$ arbitrarily in the above identity. If we choose $m=0$ 
we see immediately that equation (14) is satisfied. A dual argument (comparing second components of the two products in $\mathrm{Sp}_{4}$ and then choosing $u=0$ ) shows that (16) is satisfied. Equation (15) is of course exactly what was proved in Proposition 3.6. This completes the verification of associativity.

We denote the semigroup $(Z \times G \times Z, \cdot)$ with multiplication defined by equation (13) by $\operatorname{Sp}_{4}(G, \alpha)$. In order to show that $\operatorname{Sp}_{4}(G, \alpha)$ is a regular four-spiral semigroup we determine its idempotents and Green's relations.

LemMA 4.3. (a) The element $(m, g, n) \in \operatorname{Sp}_{4}(G, \alpha)$ is an idempotent if and only if $g=1$ (the identity of $G$ ) and $(m, n)$ is an idempotent of $\mathrm{Sp}_{4}$, i.e., $(m, n) \in E_{4}$.

(b) $(m, g, n) \Re(r, h, s)$ in $\mathrm{Sp}_{4}(G, \alpha)$ iff $m=r$.

(c) $(m, g, n) \mathcal{L}(r, h, s)$ in $\mathrm{Sp}_{4}(G, \alpha)$ iff $n=s$.

(d) $\mathrm{Sp}_{4}(G, \alpha)$ is a bisimple regular four-spiral semigroup.

Proof. It is clear that elements of the form $(m, 1, n)$ for $(m, n) *(m, n)=$ $(m, n)$ are idempotents of $\operatorname{Sp}_{4}(G, \alpha)$. Conversely if $(m, g, n) \cdot(m, g, n)=$ $(m, g, n)$, then $(m, n) *(m, n)=(m, n)$ and so $S(n, m)=(m, n)$, so by (13), $g=g \alpha^{0} g \alpha^{0}=g^{2}$, whence $g=1$. Thus (a) is satisfied. Let $(m, g, n) \in$ $\operatorname{Sp}_{4}(G, \alpha)$ and choose $p, q \in Z$ so that $(m, p) \in E_{4}$ and $(q, n) \in E_{4}$. Then $S(n, q)=(q, n)$ and so by $(13)$,

$$
(m, g, n)\left(q, g^{-1}, p\right)=\left(m, g \alpha^{0} g^{-1} \alpha^{0}, p\right)=(m, 1, p)
$$

and similarly $\left(q, g^{-1}, p\right)(m, g, n)=(q, 1, n)$, so $(m, g, n) \Re(m, 1, p)$ and $(m, g, n) \mathcal{E}(q, 1, n)$. Results (b), (c) and (d) all follow easily since it is clear that if $(m, p),(q, n) \in E_{4}$, then $(m, p) \Re[\mathcal{L}](q, n)$ in $\mathrm{Sp}_{4}$ iff $(m, 1, p) \Re[\mathcal{L}]$ $(q, 1, n)$ in $\operatorname{Sp}_{4}(G, \alpha)$. This completes the proof of the lemma and of Theorem 4.1.

We now establish the converse of Theorem 4.1.

THEOREM 4.4. If $S$ is a regular four-spiral semigroup then $S \cong \operatorname{Sp}_{4}(G, \alpha)$ for some group $G$ and endomorphism $\alpha$ of $G$.

Proof. If $\mu$ is the maximum idempotent-separating congruence on $S$ then $\mu^{\natural}: S \rightarrow S / \mu$ maps $S$ onto a fundamental four-spiral semigroup. Since $\mathrm{Sp}_{4}$ is the only fundamental four-spiral semigroup ([1, Corollary 2.11]) it follows that $S / \mu \simeq \mathrm{Sp}_{4}$, that $S$ is an $\mathcal{H}$-coextension of $\mathrm{Sp}_{4}$ and that $\mathcal{K}=\mu$ is a congruence on $S$ (because $\mathrm{Sp}_{4}$ is combinatorial).

Since $S$ is a regular four-spiral semigroup, $S$ is bisimple and so $\operatorname{tr}(S)$ consists of a single Rees groupoid $\mathfrak{N}^{0}(G ; Z, Z ; P) \backslash\{0\}$. Furthermore the subsemigroup of $S$ generated by the idempotents is a transversal subsemigroup of the $\mathcal{H}$-classes of $S$ isomorphic to $\mathrm{Sp}_{4}\left(\mathrm{Sp}_{4}\right.$ is idempotent-generated). Thus by Theorem 2.3 we may assume that $S \cong S_{1}=S(\{M\}, \Phi, \Psi)$ for $M=\mathscr{T}^{0}(G ; Z, Z ; P) \backslash\{0\}$ where $P$ is a $(0,1)$ matrix over $G^{0}$ and $\Phi$ and $\Psi$ 
are structurally uniform. Furthermore $\mathcal{H C}$ is a congruence on $S$ and $S / \mathcal{H} \cong$ $\mathrm{Sp}_{4}$ so by Corollary 1.7 the structure mappings on $S$ are $\mathcal{H}$-class preserving and commute with $\mathcal{F C}^{\natural}$ and the corresponding structure mappings on $\mathrm{Sp}_{4}$ in the sense of Theorem 1.3.

From Proposition 3.4 and Theorem 1.8 it follows that if $R_{m}>R_{r}$ and $L_{n}>L_{s}$ in $\mathrm{Sp}_{4}$ then there is just one structure mapping $\phi_{m, r}: R_{m} \rightarrow R_{r}$ and just one structure mapping $\psi_{n, s}: L_{n} \rightarrow L_{s}$. We use the same notation for the corresponding structure mappings in $S_{1}$, since no confusion can arise. Thus

$$
S_{1}=\{(m, g, n) \mid m, n \in Z, g \in G\}
$$

and if $(m, n) \phi_{m, r}=(r, p)$ and $(m, n) \psi_{n, s}=(q, s)$ in $\mathrm{Sp}_{4}$, then there exist $f, h \in G$ such that $(m, g, n) \phi_{m, r}=(r, h, p)$ and $(m, g, n) \psi_{n, s}=(q, f, s)$.

Define $\alpha: G \rightarrow G$ by $(0, g, 0) \phi_{0,1}=(1, g \alpha, 1)$. By Theorem 2.1, $(0, g, 0) \psi_{0,1}$ $=(1, g \alpha, 1)$ and $\alpha$ is an endomorphism of $G$. By structural uniformity of $\Phi$ and $\Psi$ and equation (11) one easily sees that if $R_{m} \geqslant R_{p}, L_{n}>L_{q}$ and $|p-m|=|q-n|=1$, then

$(m, g, n) \phi_{m, p}=(p, g \alpha, n+\sigma(n))$ and $(m, g, n) \psi_{n, q}=(m+\sigma(m), g \alpha, q)$.

From (k1) of [13] it follows easily that $\phi_{m, p} \phi_{p, t}=\phi_{m, t}$ for $R_{m} \geqslant R_{p} \geqslant R_{t}$ and $\psi_{n, q} \psi_{q, s}=\psi_{n, s}$ for $L_{n} \geqslant L_{q} \geqslant L_{s}$, and hence if $R_{m} \geqslant R_{p}$ and $L_{n} \geqslant L_{q}$ we have, by (11),

$$
(m, g, n) \phi_{m, p}=\left(p, g \alpha^{|p-m|}, n+\sigma(n)|p-m|\right)
$$

and

$$
(m, g, n) \psi_{n, q}=\left(m+\sigma(m)|q-n|, g \alpha^{|q-n|}, q\right) .
$$

Now let $(m, g, n),(r, h, s) \in S_{1}$ and let $(p, q)=S(n, r)$. Then by equation (2),

$$
\begin{aligned}
(m, g, n)(r, h, s) & =(m, g, n) \psi_{n, q}(r, h, s) \phi_{r p p} \\
= & \left(m+\sigma(m)|q-n|, g \alpha^{|q-n|}, q\right)\left(p, h \alpha^{|p-r|}, s+\sigma(s)|p-r|\right) \\
= & \left(m+\sigma(m)|q-n|, g \alpha^{|q-n|} h \alpha^{|p-r|}, s+\sigma(s)|p-r|\right)
\end{aligned}
$$

since $p_{q p}=1$. Thus $S_{1}=\operatorname{Sp}_{4}(G, \alpha)$ and $S \cong \operatorname{Sp}_{4}(G, \alpha)$ as required.

Theorems 4.1 and 4.4 give a description of the structure of regular fourspiral semigroups analogous to Reilly's description [23] of the structure of bisimple $\omega$-semigroups. We use Reilly's notation $S(G, \alpha)$ for the general bisimple $\omega$-semigroup in the following theorem. We also use the notation of [1] for the subsemigroups $A, B, C$ and $D \cup E$ of $\mathrm{Sp}_{4}$.

THEOREM 4.5. For each group $G$ and endomorphism $\alpha$ of $G$

(a) $\operatorname{Sp}_{4}(G, \alpha)$ is a pseudo-inverse semigroup; for each $e \in E\left(\operatorname{Sp}_{4}(G, \alpha)\right)$, $e \mathrm{Sp}_{4}(G, \alpha) e$ is a bisimple $\omega$-semigroup isomorphic to $S(G, \alpha)$; 
(b) define $\bar{A}=\left\{(m, g, n) \in \operatorname{Sp}_{4}(G, \alpha) \mid(m, n) \in A\right\}$ and similarly define $\bar{B}$, $\bar{C}, \bar{D}$ and $\bar{E}$ : then $\bar{A}, \bar{B}, \bar{C}$ and $\bar{D}$ are subsemigroups of $\operatorname{Sp}_{4}(G, \alpha)$ isomorphic to $S(G, \alpha)$ and $\bar{E}$ is a subsemigroup isomorphic to the semigroup $(N \times G, \cdot)$ with multiplication $\cdot$ defined by

$$
(m, g) \cdot(n, h)=\left(m+n, g \alpha^{n} h\right)
$$

furthermore, $\operatorname{Sp}_{4}(G, \alpha)$ is the rectangular band of $\bar{A}, \bar{B}, \bar{C}$ and $\bar{D} \cup \bar{E}$ pictured in Diagram 3.

\begin{tabular}{|r|c|}
\hline $\bar{A}$ & $\bar{B}$ \\
\hline $\bar{D} \cup \bar{E}$ & $\bar{C}$ \\
\hline
\end{tabular}

$$
\mathrm{Sp}_{4}(G, \alpha)=\bar{A} \cup \bar{B} \cup \bar{C} \cup \bar{D} \cup \bar{E}
$$

\section{Diagram 3}

(c) $\mathrm{Sp}_{4}(G, \alpha)$ is idempotent-generated iff $G=\{1\}$ (in which case $\mathrm{Sp}_{4}(G, \alpha)$ $\left.\simeq \mathrm{Sp}_{4}\right)$.

Proof. By the results of [1] we can think of $\mathrm{Sp}_{4}$ as an idempotent-generated subsemigroup of $\operatorname{Sp}_{4}(G, \alpha)$ generated by the idempotents $a=(0,1,0), b=$ $(0,1,-1), c=(-1,1,-1)$ and $d=(-1,1,1)$. Result (c) is thus immediate. Since in $\mathrm{Sp}_{4}, A=(0,0) \mathrm{Sp}_{4}(0,0)$ we see that $\bar{A}=a \mathrm{Sp}_{4}(G, \alpha) a$ : the map $\theta$ : $\bar{A} \rightarrow S(G, \alpha)$ defined by $(m, g, n) \theta=(m, g, n) \forall(m, g, n) \in \bar{A}$ is clearly a bijection from $\bar{A}$ onto $S(G, \alpha)$. Furthermore, if $(m, g, n),(r, h, s) \in \bar{A}$ then $S(n, r)=(n \vee r, n \vee r)$ by Proposition 3.2, so in $\operatorname{Sp}_{4}(G, \alpha)$

$(m, g, n)(r, h, s)=\left(m+(n \vee r)-n, g \alpha^{(n \vee r)-n} h \alpha^{(n \vee r)-r}, s+(n \vee r)-r\right)$, and this is the same as Reilly's form [23] of the multiplication in $S(G, \alpha)$. Thus $\theta$ is an isomorphism from $\bar{A}=a \mathrm{Sp}_{4}(G, \alpha) a$ onto $S(G, \alpha)$.

For each $x \in S=\operatorname{Sp}_{4}(G, \alpha)$ we denote the right translation $z \rightarrow z x$ of $S$ by $\rho_{x}$ and the left translation $z \rightarrow x z$ by $\lambda_{x}$ : one easily checks that $\bar{B}=b S b$, $\bar{C}=c S c, \bar{D}=d S d$ and that the maps $\left.\rho_{b}\right|_{\bar{A}}: \bar{A} \rightarrow \bar{B},\left.\lambda_{c}\right|_{\bar{B}}: \bar{B} \rightarrow \bar{C}$ and $\left.\rho_{d}\right|_{\bar{C}}:$ $\bar{C} \rightarrow \bar{D}$ are isomorphisms, so that $\bar{A} \simeq \bar{B} \cong \bar{C} \cong \bar{D} \cong \bar{S}(G, \alpha)$. The map

$$
(-m, g, 0) \mapsto(m, g) \quad(m \in N)
$$

is easily seen to be a bijection from $\bar{E}$ onto $N \times G$. Furthermore, if $(-m, g, 0),(-n, h, 0) \in \bar{E}$ then by Proposition 3.2, $S(0,-n)=(-n, n)$, so by (13)

$$
\begin{aligned}
(-m, g, 0)(-n, h, 0) & =\left(-m+\sigma(-m)|n|, g \alpha^{|n|} h \alpha^{|0|}, 0+\sigma(0)|0|\right) \\
& =\left(-m-n, g \alpha^{n} h, 0\right),
\end{aligned}
$$

so $\bar{E} \simeq(N \times G, \cdot)$. Result (b) now follows easily by considering the homomorphism $\phi:(m, g, n) \mapsto(m, n)$ from $\mathrm{Sp}_{4}(G, \alpha)$ onto $\mathrm{Sp}_{4}$, since $\bar{A}=A \phi^{-1}$, $\bar{B}=B \phi^{-1}, \bar{C}=C \phi^{-1}$ and $\bar{D} \cup \bar{E}=(D \cup E) \phi^{-1}$. 
The semigroup $S=\operatorname{Sp}_{4}(G, \alpha)$ is easily seen to be pseudo-inverse by the results of Nambooripad [21] since sandwich sets in $E_{4}$ are trivial. Now $E_{4}$ is a union of four $\omega$-chains,

$$
E_{4}=\left\{a_{n}, b_{n}, c_{n}, d_{n} \mid n>0\right\}=\omega(a) \cup \omega(b) \cup \omega(c) \cup \omega(d) .
$$

Part (a) of the theorem is proved by induction on $n$ : the mappings

$$
\begin{aligned}
& \left.\rho_{b_{n}}\right|_{\left(a_{n} S a_{n}\right)}: a_{n} S a_{n} \rightarrow b_{n} S b_{n},\left.\quad \lambda_{c_{n}}\right|_{\left(b_{n} S b_{n}\right)}: b_{n} S b_{n} \rightarrow c_{n} S c_{n}, \\
& \left.\rho_{d_{n}}\right|_{\left(c_{n} S c_{n}\right)}: c_{n} S c_{n} \rightarrow d_{n} S d_{n} \text {, and }\left.\lambda_{a_{n}}\right|_{\left(d_{n} S d_{n}\right)}: d_{n} S d_{n} \rightarrow a_{n+1} S a_{n+1}
\end{aligned}
$$

are easily seen to be isomorphisms. Thus, by induction on $n, e S e \simeq S(G, \alpha)$ for each $e \in E(S)=E\left(\operatorname{Sp}_{4}(G, \alpha)\right)$.

5. The isomorphism theorem. In this section we determine conditions under which $\operatorname{Sp}_{4}\left(G_{1}, \alpha_{1}\right) \cong \operatorname{Sp}_{4}\left(G_{2}, \alpha_{2}\right)$. Recall that in [23] Reilly proved that there is an isomorphism $\phi$ from $S\left(G_{1}, \alpha_{1}\right)$ onto $S\left(G_{2}, \alpha_{2}\right)$ if and only if there is an isomorphism $\theta$ of $G_{1}$ onto $G_{2}$ such that $\alpha_{1} \theta=\theta \alpha_{2} T_{2}$ where, for some element $z$ of $G_{2}, T_{z}$ is the inner automorphism of $G_{2}$ defined by $g T_{z}=z g z^{-1}$ : the mapping $\theta$ is defined from $\phi$ by $(0, g, 0) \phi=(0, g \theta, 0)$ and the element $z$ is obtained from the equation $(0,1,1) \phi=(0, z, 1)$. We assume familiarity with Reilly's results and we obtain a corresponding result for the semigroups $\mathrm{Sp}_{4}(G, \alpha)$.

TheOREM 5.1. There is an isomorphism $\phi$ from $\operatorname{Sp}_{4}\left(G_{1}, \alpha_{1}\right)$ onto $\operatorname{Sp}_{4}\left(G_{2}, \alpha_{2}\right)$ (for groups $G_{i}$ and endomorphisms $\alpha_{i}$ of $\left.G_{i}, i=1,2\right)$ if and only if there is an isomorphism $\theta$ from $G_{1}$ onto $G_{2}$ such that $\alpha_{1} \theta=\theta \alpha_{2}$.

Proof. Suppose first that $\phi$ is an isomorphism from $\operatorname{Sp}_{4}\left(G_{1}, \alpha_{1}\right)$ onto $\mathrm{Sp}_{4}\left(G_{2}, \alpha_{2}\right)$. Denote the subsemigroups of $\operatorname{Sp}_{4}\left(G_{i}, \alpha_{i}\right)(i=1,2)$ defined in Theorem 4.5 by $\bar{A}_{i}, \bar{B}_{i}, \bar{C}_{i}, \bar{D}_{i}$ and $\bar{E}_{i}(i=1,2)$. The isomorphism $\phi$ induces a biorder isomorphism from

$$
E\left(\operatorname{Sp}_{4}\left(G_{1}, \alpha_{1}\right)\right)=\left\{a_{n}^{(1)}, b_{n}^{(1)}, c_{n}^{(1)}, d_{n}^{(1)} \mid n \in N\right\}
$$

onto

$$
E\left(\operatorname{Sp}_{4}\left(G_{2}, \alpha_{2}\right)\right)=\left\{a_{n}^{(2)}, b_{n}^{(2)}, c_{n}^{(2)}, d_{n}^{(2)} \mid n \in N\right\}
$$

and under this biorder isomorphism we have $a_{n}^{(1)} \rightarrow a_{n}^{(2)}, b_{n}^{(1)} \rightarrow b_{n}^{(2)}, c_{n}^{(1)} \rightarrow c_{n}^{(2)}$, $d_{n}^{(1)} \rightarrow d_{n}^{(2)} \forall n \in N$. Now

$$
E\left(\bar{A}_{i}\right)=\left\{a_{n}^{(i)} \mid n \in N\right\} \quad(\text { for } i=1,2) ;
$$

if $x \in \overline{A_{1}}$ then $x \Re a_{n}^{(1)}$ and $x \mathcal{E} a_{m}^{(1)}$ for some $n, m \in N$, so $x \phi \Re a_{n}^{(2)}$ and $x \phi \subseteq a_{m}^{(2)}$ in $\overline{A_{2}}$, and it follows that $\bar{A}_{1} \phi \subseteq \bar{A}_{2}$. Dually, $\bar{A}_{2} \phi^{-1} \subseteq \overline{A_{1}}$, so $\bar{A}_{1} \phi=\bar{A}_{2}$, i.e., $\phi$ induces an isomorphism from $\bar{A}_{1}$ onto $\overline{A_{2}}$. But by Theorem 4.5 we may identify $\bar{A}_{i}$ and $S\left(G_{i}, \alpha_{i}\right)(i=1,2)$ and so there is an isomorphism 
(which we continue to denote by $\phi$ ) from $S\left(G_{1}, \alpha_{1}\right)=A_{1}$ onto $S\left(G_{2}, \alpha_{2}\right)=$ $A_{2}$. By the results of Reilly [23], we have $\alpha_{1} \theta=\theta \alpha_{2} T_{z}$ where $\theta, z$ and $T_{z}$ are as described in the discussion above: we aim to show that $z=1$ (the identity of $G_{1}$ ), from which it follows that $\alpha_{1} \theta=\theta \alpha_{2}$ as required.

Note first that a minor variation of the argument given above to show that $\overline{A_{1}} \phi \subseteq \overline{A_{2}}$ establishes that if $(m, g, n) \in \operatorname{Sp}_{4}\left(G_{1}, \alpha_{1}\right)$ then $(m, g, n) \phi=$ $(m, h, n)$ for some $h \in G_{2}$. Consider a product in $\operatorname{Sp}_{4}\left(G_{1}, \alpha_{1}\right)$ of the form $(m, g, n)(n, 1,-(n+1))$ where $m<0$ and $n \geqslant 0$. This is a product in the trace, so

$$
(m, g, n)(n, 1,-(n+1))=(m, g,-(n+1))
$$

and so

$$
(m, g,-(n+1)) \phi=(m, g, n) \phi(n, 1,-(n+1)) \phi .
$$

But $(n, 1,-(n+1))=b_{n}^{(1)}$ so $(n, 1,-(n+1)) \phi=b_{n}^{(2)}$. It follows that $(m, g, n) \phi=\left(m, g_{1}, n\right)$ iff $(m, g,-(n+1)) \phi=\left(m, g_{1},-(n+1)\right)$. By considering the products $(0,1,0)(m, g, n)$ and $(0,1,-1)(m, g,-(n+1))(0,1,0)$ and applying the homomorphism $\phi$ one sees that

$$
(m, g, n) \phi=\left(m, g_{1}, n\right) \text { iff }(-m, g, n) \phi=\left(-m, g_{1}, n\right)
$$

and

$$
\begin{aligned}
(m, g,-(n+1)) \phi & =\left(m, g_{1},-(n+1)\right) \\
& \text { iff }(-(m+1), g, n) \phi=\left(-(m+1), g_{1}, n\right) .
\end{aligned}
$$

Hence $(-m, g, n) \phi=\left(-m, g_{1}, n\right)$ iff $(-(m+1), g, n) \phi=\left(-(m+1), g_{1}, n\right)$, for all $m<0$ and $n \geqslant 0$. Hence

$$
(p, g, n) \phi=\left(p, g_{1}, n\right) \text { iff }(p+1, g, n) \phi=\left(p+1, g_{1}, n\right)
$$

for all $p>0$ and $n \geqslant 0$. In particular, choosing $p=n=0$ and $g=1$ we see that $(1,1,0) \phi=(1,1,0)$ (since $(0,1,0) \phi=(0,1,0))$. But $(0,1,1) \phi=(0, z, 1)$ and $(1,1,0)$ is the inverse of $(0,1,1)$ in $S\left(G_{1}, \alpha_{1}\right)$, so $(1,1,0) \phi=\left(1, z^{-1}, 0\right)$, from which it follows that $z=1$ as required.

Suppose conversely that there is an isomorphism $\theta$ from $G_{1}$ onto $G_{2}$ such that $\alpha_{1} \theta=\theta \alpha_{2}$ and consider the mapping $\phi$ : $\operatorname{Sp}_{4}\left(G_{1}, \alpha_{1}\right) \rightarrow \operatorname{Sp}_{4}\left(G_{2}, \alpha_{2}\right)$ defined by $(m, g, n) \phi=(m, g \theta, n)$. The mapping $\phi$ is clearly a trace isomorphism since $\theta$ is a group isomorphism. It remains to show that $\phi$ commutes with the structure mappings in the sense of Theorem 1.3. If, in $\operatorname{Sp}_{4}\left(G_{1}, \alpha_{1}\right)$, $R_{m}>R_{p}$ then

$$
\begin{aligned}
(m, g, n) \phi_{m, p}^{(1)} \phi & =\left(p, g \alpha_{1}^{|p-m|}, n+\sigma(n)|p-m|\right) \phi \\
& =\left(p, g \alpha_{1}^{|p-m|} \theta, n+\sigma(n)|p-m|\right) \\
& =\left(p, g \theta \alpha_{2}^{|p-m|}, n+\sigma(n)|p-m|\right) \\
& =(m, g \theta, n) \phi_{m, p}^{(2)}=(m, g, n) \phi \phi_{m, p}^{(2)}
\end{aligned}
$$


A similar argument shows that $\phi$ commutes with the $\psi$-mappings. This completes the proof of the theorem.

As an immediate corollary we have the following:

COROllary 5.2. The semigroup $\mathrm{Sp}_{4}(G, \alpha)$ is isomorphic to the direct product of $\mathrm{Sp}_{4}$ and $G$ if and only if $\alpha=\imath$, the identity automorphism of $G$.

REMARK 5.3. Theorem 5.1 shows that not every isomorphism from $S\left(G_{1}, \alpha_{1}\right)$ onto $S\left(G_{2}, \alpha_{2}\right)$ extends to an isomorphism from $\operatorname{Sp}_{4}\left(G_{1}, \alpha_{1}\right)$ onto $\mathrm{Sp}_{4}\left(G_{2}, \alpha_{2}\right)$ : for example if $\alpha$ is an inner automorphism of $G$ then by Corollary 4.2 of [23], $S(G, \alpha) \cong S(G, \imath) \cong G \times C(p, q)$, where $\mathcal{C}(p, q)$ is the bicyclic semigroup ([3, vol. 1, p. 43]), but if $\alpha \neq \imath$ there is no automorphism $\theta$ of $G$ such that $\alpha \theta=\theta \iota=\theta$, and so $\operatorname{Sp}_{4}(G, \alpha)$ is not isomorphic to $\operatorname{Sp}_{4}(G, \iota)=$ $\mathrm{Sp}_{4} \times G$.

ACKnowledgements. The author wishes to thank K. Byleen and N. R. Reilly for several stimulating conversations and suggestions.

\section{REFERENCES}

1. K. Byleen, J. Meakin and F. Pastijn, The fundamental four-spiral semigroup, J. Algebra 54 (1978), 6-26.

2. A. H. Clifford, The fundamental representation of a regular semigroup, Math. Dept. Tulane Univ. (July 1974); announced in Semigroup Forum 10 (1975), 84-92.

3. A. H. Clifford and G. B. Preston, The algebraic theory of semigroups, vols. I, II, Math. Surveys, No. 7, Amer. Math. Soc., Providence, R. I., $1961,1967$.

4. A. Coudron, Sur les extensions de demi-groupes réciproques, Bull. Soc. Roy. Sci. Liège 37 (1968), 409-419.

5. H. D'Alarcao, Idempotent-separating extensions of inverse semigroups, J. Austral. Math. Soc. 9 (1969), 211-217.

6. P. A. Grillet, The structure of regular semigroups. III, The reduced case, Semigroup Forum 8 (1974), 266-269.

7. The structure of regular semigroups. IV, The general case, Semigroup Forum 8 (1974), 368-373.

8. T. E. Hall, On orthodox semigroups and uniform and anti-uniform bands, J. Algebra 16 (1970), 204-217.

9. On regular semigroups, J. Algebra 24 (1973), 1-24.

10. G. Lallement et M. Petrich, Décompositions I-matricielles d'un demi-groupe, J. Math. Pures Appl. 45 (1966), 67-117.

11. J. E. Leech, $\mathcal{H}$-coextensions and the structure of bands of groups, Mem. Amer. Math. Soc. No. 157 (1975).

12. J. Meakin, On the structure of inverse semigroups, Semigroup Forum 12 (1976), 6-14.

13. The structure mappings on a regular semigroup, Proc. Edinburgh Math. Soc. 21 (1978), 135-142.

14. __ Coextensions of inverse semigroups, J. Algebra 46 (1977), 315-333.

15. W. D. Munn, Uniform semilattices and bisimple inverse semigroups, Quart. J. Math. 17 (1966), 151-159.

16. __ Fundamental inverse semigroups, Quart. J. Math. 21 (1970), 157-170.

17. __ Some results on 0-simple inverse semigroups, Proc. Sympos. on Inverse Semigroups, Northern Illinois Univ., Feb. 1973.

18. K. S. S. Nambooripad, Structure of regular semigroups, Thesis, Univ. of Kerala, Sept. 1973. 
19. Structure of regular semigroups. I, Fundamental regular semigroups, Semigroup Forum 9 (1975), 354-363.

20. $364-371$.

21. __ Pseudo-semilattices and biordered sets, Proc. Edinburgh Math. Soc. (submitted).

22. _ The natural partial order on a regular semigroup, Proc. Edinburgh Math. Soc. (submitted).

23. N. R. Reilly, Bisimple w-semigroups, Proc. Glasgow Math. Assoc. 7 (1960), 160-167.

24. B. M. Schein, Pseudo-semilattices and pseudo-lattices, Izv. Vysš. Učebn. Zaved. Matematika 1972, no. 2 (117), 81-94. (Russian)

Departaknt of Mathematics \& Statistics, Universtty of Nebrasta, LnNColn, Nebrastea 68588 